\title{
Magnetopolaron effect on shallow-impurity states in the presence of magnetic and intense terahertz laser fields in the Faraday configuration
}

\author{
Weiyang Wang, ${ }^{1,2}$ B. Van Duppen, ${ }^{2, *}$ M. Van der Donck, ${ }^{2}$ and F. M. Peeters ${ }^{2, \dagger}$ \\ ${ }^{1}$ School of Physics and Electronics Information, Shangrao Normal University, Shangrao, Jiangxi 334001, People's Republic of China \\ ${ }^{2}$ Departement Fysica, Universiteit Antwerpen Groenenborgerlaan 171, B-2020 Antwerpen, Belgium
}

(Received 13 December 2017; published 26 February 2018)

\begin{abstract}
The magnetopolaron effect on shallow-impurity states in semiconductors is investigated when subjected simultaneously to a magnetic field and an intense terahertz laser field within the Faraday configuration. We use a time-dependent nonperturbative theory to describe electron interactions. The externally applied fields are exactly included via a laser-dressed interaction potential. Through a variational approach we evaluate the binding energy of the shallow-impurity states. We find that the interaction strength of the laser-dressed Coulomb potential can not only be enhanced but also weakened by varying the two external fields. In this way, the binding energy can be tuned by the external fields and red- or blue-shifted with respect to the static binding energy. In the nonresonant polaron region, a magnetopolaron correction that includes the effects of photon process is observed. In the resonant polaron region, moreover, the resonant magnetopolaron effect accompanied by the emission and absorption of a single photon is distinctly observed. This can be modulated to be far away from the reststrahlen band. The intriguing findings of this paper can be observed experimentally and, in turn, provide a way to measure the strength of the electron-phonon interaction.
\end{abstract}

DOI: 10.1103/PhysRevB.97.064108

\section{INTRODUCTION}

The intriguing characteristics of shallow-impurity states in semiconductors and their nanostructures have attracted a lot of interest in the past few decades. This is due to the fact that shallow-impurity states in semiconductor systems affect many characteristics of the semiconductor such as conductivity [1], impurity cyclotron resonance [2], impurity-assisted tunneling [3], to mention a few, and also play an important role in determining the optical and transport properties of these devices at low temperature. Moreover, the behavior of shallow-impurity states under a static magnetic field can provide a deeper insight into several problems in astrophysics [4].

Nowadays, with the development of state-of-the-art laser sources such as free-electron lasers (FELs), new possibilities have arisen in probing the behavior of shallow-impurity states in semiconductors and their nanostructures when irradiated by intense terahertz (THz) laser fields (ITLFs). This is due to the fact that the applied $\mathrm{THz}$ laser field frequency is nearly of the order of characteristic frequencies in these systems, which suggests that an ITLF can intensively interact with semiconductor systems and processes of momentum and energy excitation and relaxation of electrons can be significantly modified in such laser-driven systems.

The influence of the ITLFs on shallow-impurity states in semiconductor systems can be detected, for instance, by measuring the distortion of the binding energy and intradonor transitions associated with those shallow-impurity states. This shows up as modifications of optical properties in

\footnotetext{
*ben.vanduppen@uantwerpen.be

†francois.peeters@uantwerpen.be
}

the systems under irradiation. Consequently, the behavior of shallow-impurity states in laser-driven systems has attracted the attention of research communities around the world in the last decades [5-9]. Already some interesting phenomena associated with shallow-impurity states have been obtained theoretically or experimentally; including dichotomy of hydrogen atoms [10,11] and excitons [12], shallow-impurity states in bulk semiconductors that become stable against ionization in the high-intensity limit [13], a reduction of the binding energy with increasing laser field intensity in semiconductors $[13,14]$ and their nanostructures $[15,16]$.

Moreover, it has also been shown that the effect of the ITLFs on the intraimpurity state transitions in semiconductor nanostructures can be comparable to that of a magnetic field [8]. More recently, particular interest has been paid to the nonlinear magneto-optical properties of shallow-impurity states in quantum wells [17-20], quantum well wires [21,22], and quantum dots [23] subjected simultaneously to ITLFs and magnetic fields. This interest has been motivated, in part, due to the opening of a promising route to design new efficient optoelectronic devices manipulated by two external applied fields [24]. These studies show that the electronic and optical properties of shallow-impurity states in semiconductor systems strongly depend not only on the geometric parameters and the laser-dressed Coulomb potential and quantum confinement, but also on the two external applied fields. This clearly indicates that both the laser field and magnetic field intensities can be properly chosen, together with an appropriate choice of the geometric parameters of the considered systems, to generate the desired energy spectrum for shallow-impurity states in semiconductors and related nanostructures. In addition, it has also been found that the conduction-electron $g$ factor in heterostructures [25] and the exciton binding energy in quantum 
well wires [26] in the presence of the ITLFs and magnetic fields can be effectively manipulated and tuned by changing the two externally applied fields. However, these previous studies were only focused on the Voigt configuration in which the vector potentials induced by the ITLFs and magnetic fields do not couple directly with each other. Notice that theoretical approaches used previously, i.e., the so-called "dressed-band approach" $[8,17,22,23]$ and the "dressed-atom approach" [18-21] are not valid in the Faraday configuration. Recently, we proposed a time-dependent nonperturbative approach to investigate the binding energy and transition energy for ground $1 s$-like and excited $2 p^{-}$-like states in bulk semiconductors under the ITLFs and magnetic fields within the Faraday configuration [27].

Due to the single-particle nature of the shallow impurity system, possible complications from many-body effects such as screening and Pauli exclusion effects [28] are not important in the interpretation of the observed phenomena. As a consequence, shallow impurities in polar semiconductors and related nanostructures provide a natural and almost ideal system to investigate the interaction between electrons and longitudinal-optical (LO) phonons, which has received a lot of attention in the past. The importance of this interaction not only arises from the fact that it modifies the carrier effective mass [29], but also comes from its influence on the device performance through carrier velocity and mobility. The consequences of electron-phonon (e-p) interaction are specifically apparent when the magneto-optical transition energy associated with the shallow-impurity states is tuned into resonance with the LO phonon energy by means of a high magnetic field, leading to a resonant splitting of this transition. As a result, a characteristic anticrossing behavior, so-called resonant magnetopolaron effect (RMPE), can be observed directly, which has been intensively studied in bulk semiconductors [30-32] as well as in the related nanostructures including quantum wells [33], superlattices [34], and quantum dots [35]. This resonant splitting of the shallow-impurity states in semiconductor systems in turn provides a direct measure of the e-p interaction. More interestingly, the e-p interaction in semiconductors and related nanostructures under ITLFs can be appropriately modulated by the ITLFs [36] and effectively suppressed when the laser field intensity is strong enough [37-39], while the effective electron-photon coupling is enhanced. Therefore, it is more reasonable to experimentally observe an intriguing resonant magnetopolaron effect modulated by the two external applied fields in polar semiconductors and their nanostructures subjected to the ITLFs and magnetic fields simultaneously in the Faraday configuration. However, such novel phenomena have not been investigated up to now.

In this study, we are concerned with the behavior of shallow-impurity states in polar bulk semiconductors under ITLFs and magnetic fields within the Faraday configuration. Using a nonperturbative approach and a variational method, we investigate the effect of two external applied fields, an electric field and a magnetic field, on the energy levels associated with the shallow-impurity states. A resonant magnetopolaron effect adjusted by the external applied fields is predicted using the time-dependent perturbation approach, leading to new intriguing physical phenomena. This paper is organized as follows. In Sec. II we outline the nonperturbative approach together with the time-dependent perturbation approach. Our

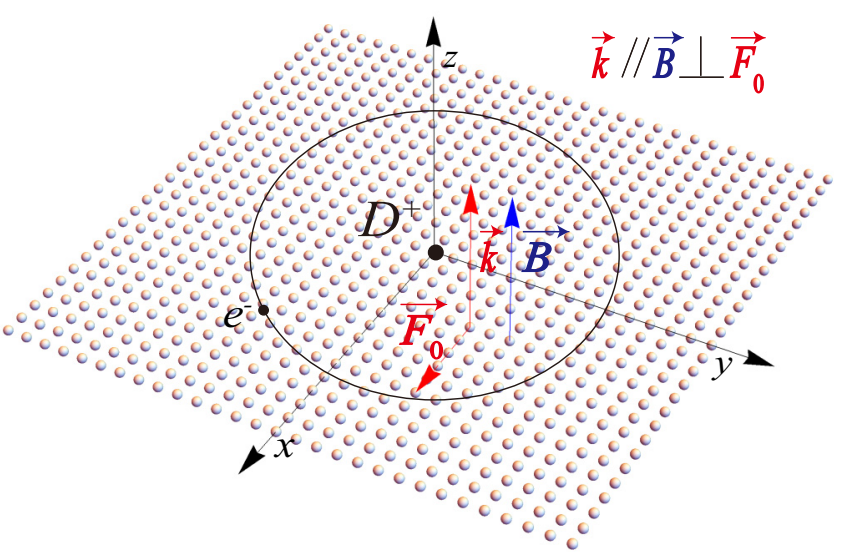

FIG. 1. Schematic representation of a shallow donor $D^{+}$in a bulk semiconductor in the presence of ITLF and magnetic field within the Faraday configuration. Here, $\vec{k}$ is the wave vector of the applied ITLF.

numerical results and discussions are presented in Sec. III and concluding remarks are given in Sec. IV.

\section{THEORETICAL APPROACH}

We consider a shallow donor impurity in a bulk semiconductor which is simultaneously subjected to an ITLF and a static magnetic field along the $z$ axis, where the laser field is linearly polarized along the $x$ axis, as shown in Fig. 1. Under the usual dipole approximation, the vector potential of the laser field is given as $\vec{A}_{t}=\left(F_{0} / \omega\right) \cos (\omega t) \vec{e}_{x}$ with $F_{0}$ and $\omega$ being, respectively, the amplitude and angular frequency of the laser field. Within the framework of the effective-mass approximation and assuming that the dielectric constant $\epsilon$ and electron effective mass $m^{*}$ are both isotropic, the total Hamiltonian for a single conduction band electron coupled to a hydrogenic donor and interacting with longitudinal-optical (LO) phonons is described by

$$
H=H_{e}(\vec{R}, t)+H_{\mathrm{LO}}+H_{I}(\vec{R}),
$$

where $H_{e}(\vec{R}, t)$ is the Hamiltonian for the hydrogenic donor electron in the two external applied fields

$$
H_{e}(\vec{R}, t)=\frac{1}{2 m^{*}}(\vec{p}+e \vec{A})^{2}+V(\vec{R}) .
$$

Here, $\vec{A}=\vec{A}_{t}+\vec{A}_{B}$ with $\vec{A}_{B}=(-e B y / 2, e B x / 2,0)$ being the vector potential induced by the magnetic field and expressed in the symmetric gauge, and $V(\vec{R})=-e^{2} / \epsilon|\vec{R}|$ is the Coulomb potential induced by the electron-impurity coupling. $\epsilon$ is the dielectric constant of the medium and $\vec{p}$ and $\vec{R}$ are the momentum and position operators of the electron, respectively. In Eq. (1), the LO phonon Hamiltonian is taken to be

$$
H_{\mathrm{LO}}=\sum_{\vec{Q}} \hbar \omega_{\vec{Q}}\left(a_{\vec{Q}}^{\dagger} a_{\vec{Q}}+\frac{1}{2}\right),
$$

where $\left(a_{\vec{Q}}^{\dagger}, a_{\vec{Q}}\right)$ are the creation and annihilation operators of a LO phonon with wave vector $\vec{Q}=\left(\vec{q}, q_{z}\right)=\left(q_{x}, q_{y}, q_{z}\right)$ and phonon frequency $\omega_{\vec{Q}}$. Finally, the Hamiltonian of the 
electron-phonon (e-p) interaction is given by

$$
H_{I}(\vec{R})=\sum_{\vec{Q}}\left(V_{\vec{Q}} a_{\vec{Q}} e^{i \vec{Q} \cdot \vec{R}}+V_{\vec{Q}}^{*} a_{\vec{Q}}^{\dagger} e^{-i \vec{Q} \cdot \vec{R}}\right) .
$$

Here, $V_{\vec{Q}}$ is the Fourier coefficient of electron-phonon coupling given by the Fröhlich Hamiltonian and taken to be $\left|V_{\vec{Q}}\right|^{2}=$ $4 \pi \alpha L_{0}\left(\hbar \omega_{\vec{Q}}\right)^{2} / \Omega Q^{2}$, where $\alpha$ is the coupling constant, $L_{0}=$ $\left(\hbar / 2 m^{*} \omega_{\vec{Q}}\right)^{1 / 2}$ is the polaron radius, and $\Omega$ is the crystal volume of the system.

\section{A. Nonperturbative approach}

Since in the Faraday configuration vector potentials induced by ITLF and magnetic field are coupled with each other, resonant phenomena can be directly observed experimentally like the cyclotron resonance effect. As a result, the dressed-atom approach [18-21] which is based on the Kramers-Henneberger (KH) unitary transformation [5,6] and the dressed-band approach $[8,17,22,23]$ are only valid in the nonresonant region and therefore cannot be used to seek the solution of the following time-dependent Schrödinger equation (TDSE) for the electronic part:

$$
i \hbar \frac{\partial \Psi(\vec{R}, t)}{\partial t}=H_{e}(\vec{R}, t) \Psi(\vec{R}, t) .
$$

On the other hand, direct numerical integration in time steps is a strenuous computational task [11]. Note that usual perturbation theory is far beyond the limit of its validity for large laser field intensities, so only nonperturbative approaches should be attempted.

Thus, we have to develop a new nonperturbative approach to deal with such a time-dependent problem, namely, one in which the laser field can be treated in a more exact way. Following the nonperturbative approach proposed previously [27,39,40], we use the time-dependent unitary transformation [27]

$$
\begin{aligned}
U= & \exp \left(\frac{i}{\hbar} f_{t}\right) \exp \left[\frac{i}{\hbar}\left(u_{t} x+v_{t} y\right)\right] \exp \left(\frac{i}{\hbar} x_{t} p_{x}\right) \\
& \times \exp \left(\frac{i}{\hbar} y_{t} p_{y}\right),
\end{aligned}
$$

where $p_{x}=-i \hbar \partial / \partial x$ is the momentum operator along the $x$ direction, the coordinate shifts $x_{t}$ and $y_{t}$ produce a translation in space, the phase shifts $u_{t}$ and $v_{t}$ lead to a translation in momentum, and $f_{t}$ takes care of the terms in the Hamiltonian depending only on time. In such a case, the electronic wave function $\Psi(\vec{R}, t)$ can be expressed as

$$
\Psi(\vec{R}, t)=U \psi(\vec{R}, t) .
$$

By inserting Eqs. (6) and (7) into Eq. (5), one obtains

$$
i \hbar \frac{\partial \psi(\vec{R}, t)}{\partial t}=\widetilde{H}_{e}(\vec{R}, t) \psi(\vec{R}, t),
$$

where

$$
\widetilde{H}_{e}(\vec{R}, t)=\frac{1}{2 m^{*}}\left(\vec{p}+e \vec{A}_{B}\right)^{2}+V[\vec{R}-\vec{c}(t)],
$$

with $\vec{c}(t)=x_{t} \vec{e}_{x}+y_{t} \vec{e}_{y}$. Here, $\vec{c}(t)$ describes the quiver motion of the classical electron under the two external applied fields, which is significantly different from the back-and-forth motion along the polarized direction of the applied ITLF in the absence of magnetic field [5-7]. Moreover, Eq. (8) characterizes the dynamics of shallow-impurity states in semiconductor systems and produces an intriguing behavior when irradiated by the two external fields, in which the electrons interact with the oscillating potential $V[\vec{R}-\vec{c}(t)]$. This is because the time dependence has been transferred from the momentum term to the interaction potential in this accelerated frame of reference. In addition, no assumptions concerning the validity of Eq. (8) in the accelerated frame of reference has been made so far, so that Eqs. (5) and (8) must be completely equivalent.

Due to the arbitrariness of the functions $x_{t}, y_{t}, u_{t}, v_{t}$, and $f_{t}$, one can use them to cancel the time-dependent terms in $\widetilde{H}_{e}(\vec{R}, t)$ that are linear in $\vec{p}$ and $\vec{R}$ and the terms that depend only on time [40]. Note that such initial conditions have been widely and successfully used in solving time-dependent problems [41,42]. In doing so and performing some algebraic manipulations, the shifts of coordinates are obtained as

$$
x_{t}=r_{0} \sin (\omega t)
$$

and

$$
y_{t}=-r_{0} \eta \cos (\omega t),
$$

where $r_{0}=e F_{0} /\left[m^{*}\left(\omega_{c}^{2}-\omega^{2}\right)\right]$ and $\eta=\omega_{c} / \omega$ with $\omega_{c}=e B / m^{*}$ being the cyclotron frequency. In addition, the phase shifts are given by $v_{t}=-r_{1} \sin (\omega t)$ and $u_{t}=-r_{1} \eta \cos (\omega t) \quad$ with $\quad r_{1}=e F_{0} \omega_{c} /\left[2\left(\omega_{c}^{2}-\omega^{2}\right)\right]$, and $f_{t}=-E_{e m}[t+\sin (2 \omega t) / 2 \omega]$ with an energy $E_{\text {em }}=$ $e^{2} F_{0}^{2} /\left[4 m^{*}\left(\omega^{2}-\omega_{c}^{2}\right)\right]$ induced by the two external applied fields. More interestingly, $E_{e m}$ will result in a shift in the fundamental absorption edge known as magneto-optical dynamical Franz-Keldysh effect (MODFKE) [43] in semiconductor systems under ITLFs and magnetic fields, which can be reduced to the ponderomotive energy $E_{e m}=e^{2} F_{0}^{2} /\left(4 m^{*} \omega^{2}\right)$ in the absence of magnetic field, leading to the usual dynamical Franz-Keldysh effect in a laser-driven semiconductor system.

\section{Laser-dressed Coulomb potential}

The explicit expression of the laser-dressed Coulomb potential $(\mathrm{LdCP})$ is given by

$$
V[\vec{R}-\vec{c}(t)]=-\frac{e^{2}}{\epsilon\left(R^{2}+a^{2}\right)^{1 / 2}}[1-b(t)]^{-1 / 2},
$$

where $b(t)=\left[a^{2} \eta_{0} \cos (2 \omega t)+2 \vec{R} \cdot \vec{c}(t)\right] /\left(R^{2}+a^{2}\right) \quad$ with $\eta_{0}=\left(1-\eta^{2}\right) /\left(1+\eta^{2}\right)$ and $a=\left|r_{0}\right|\left[\left(1+\eta^{2}\right) / 2\right]^{1 / 2}$. By application of the previous approximation method [44], Eq. (10) can be cast into a new one which is essentially the time average of the LdCP, namely,

$$
V(R ; a)=-\frac{e^{2}}{\epsilon\left(R^{2}+a^{2}\right)^{1 / 2}}\left[1+\frac{3}{16} \frac{8 R^{2} a^{2}+a^{4} \eta_{0}^{2}}{\left(R^{2}+a^{2}\right)^{2}}\right],
$$

which is quite similar to the well-known results obtained in Ref. [45]. Note that this adopted approximation has no bearing as to the laser field strength $[44,45]$, which indicates that Eq. (11) is valid for the description of shallow-impurity states in our considered systems under either an intense or a weak laser field. Moreover, this approximation has been widely used in atomic and molecular problems $[45,46]$ and successfully 
extended to study the physical properties of shallow-impurity states in semiconductor nanostructures under ITLFs $[16,47]$. In the absence of the ITLFs, the LdCP returns back to the bare Coulomb potential. On the contrary, the LdCP approaches zero in the high laser field limit or in the vicinity of a cyclotron frequency, which implies that the shallow-impurity states in semiconductor systems under the two external fields can be tuned into unbound states by changing both external fields.

\section{Laser-dressed potential for electron-phonon coupling}

In the accelerated frame of reference, the Hamiltonian $H_{I}(\vec{R})$ for e-p interaction has also been transformed into a laser-dressed oscillating potential [36,39]. This can be obtained by $U^{\dagger} H_{I}(\vec{R}) U$, namely,

$$
\widetilde{H}_{I}(\vec{R}, t)=\sum_{\vec{Q}, n}\left[\widetilde{V}_{\vec{Q}} a_{\vec{Q}} e^{i(\vec{Q} \cdot \vec{R}+n \theta)}+\widetilde{V}_{\vec{Q}}^{*} a_{\vec{Q}}^{\dagger} e^{-i(\vec{Q} \cdot \vec{R}+n \theta)}\right],
$$

where $\widetilde{V}_{\vec{Q}}=\left(\chi_{q} / \chi_{q}^{*}\right)^{n / 2} J_{n}[Z(q)] V_{\vec{Q}}$ is the laser-modulated e-p coupling with $\chi_{q}=\eta q_{y}+i q_{x}, \theta=\omega t$, and $J_{n}(x)$ is the Bessel function of the first kind with $Z(q)=\left|r_{0}\right|\left(\chi_{q} \chi_{q}^{*}\right)^{1 / 2}$. Equation (12) shows that the laser-dressed interaction potential [LdIP, i.e., $\left.\widetilde{H}_{I}(\vec{R}, t)\right]$ depends explicitly on time, so that it cannot produce a stationary e-p interaction, particulary in the high-frequency limit. As a result, the electron sees a LdIP induced by the two external applied fields, namely, the electron motion is dominated by the oscillation stemming from the ITLFs. In the absence of the ITLFs (i.e., $F_{0}=0$ ), due to $\lim _{x \rightarrow 0} J_{n}(x)=\delta_{n, 0}, \widetilde{H}_{I}(\vec{R}, t)$ goes back to the bare one, namely, $H_{I}(\vec{R})$. In contrast, in the opposite limit of intense laser field intensity (i.e., $F_{0} \rightarrow \infty$ ) or in the vicinity of $\omega_{c}$ (i.e., $\omega \rightarrow \omega_{c}$ ) the Bessel function in Eq. (12) approaches zero since $\lim _{x \rightarrow \infty} J_{n}(x)=0$. Consequently, an increase of laser field intensity (or as $\omega$ approaches $\omega_{c}$ and vice versa) leads to a weakening of the laser-dressed e-p interaction. This has been observed experimentally $[37,38]$ and investigated theoretically $[38,39]$. Most importantly, it has been explicitly confirmed that $\widetilde{H}_{I}(\vec{R}, t)$ can be reasonably treated as a perturbation potential in studying the intriguing properties of semiconductor systems under ITLFs $[38,39]$.

\section{B. Electronic states}

By inserting Eq. (11) into Eq. (9), the time-dependent Hamiltonian $\breve{H}_{e}(\vec{R}, t)$ transfers into a practically timeindependent one, namely,

$$
\widetilde{H}_{e}(\vec{R})=\frac{1}{2 m^{*}}\left(\vec{p}+e \vec{A}_{B}\right)^{2}+V(R ; a) .
$$

As a result, we can seek stationary solutions of Eq. (13) in the accelerated frame of reference to investigate the characteristics of shallow-impurity states in generic hydrogenic systems under the two external applied fields. In this case, the corresponding solutions can be expressed as $\psi_{i}(\vec{R}, t)=e^{-i E_{i}^{(0)} t / \hbar} \psi_{i}(\vec{R})$, where the wave function $\psi_{i}(\vec{R})$ and the energy spectrum $E_{i}^{(0)}$ are determined by $\left[\widetilde{H}_{e}(\vec{R})-E_{i}^{(0)}\right] \psi_{i}(\vec{R})=0$.

By considering the symmetry of our system, we chose the cylindrical polar coordinate system $(\rho, \phi, z)$ and introduce dimensionless units expressed in terms of the Bohr radius $a_{0}=$ $\hbar^{2} \epsilon / m^{*} e^{2}$ and the effective Rydberg energy $R^{*}=\hbar^{2} / 2 m^{*} a_{0}^{2}$, and then Eq. (13) can be rewritten as

$$
\begin{aligned}
\widetilde{H}_{e}(\vec{R})= & -\left[\frac{\partial^{2}}{\partial \rho^{2}}+\frac{1}{\rho} \frac{\partial}{\partial \rho}+\frac{1}{\rho^{2}} \frac{\partial^{2}}{\partial \varphi^{2}}+\frac{\partial^{2}}{\partial z^{2}}\right]+\frac{1}{4} \gamma^{2} \rho^{2} \\
& +\gamma L_{z}-\frac{2}{\sqrt{R^{2}+a^{\prime 2}}}\left[1+\frac{3}{16} \frac{8 R^{2} a^{\prime 2}+a^{\prime 4} \eta_{0}^{2}}{\left(R^{2}+a^{\prime 2}\right)^{2}}\right],
\end{aligned}
$$

where $L_{z}=-i(\partial / \partial \varphi)$ is the $z$ component of the angular momentum operator, $\gamma=a_{0}^{2} / l_{B}^{2}$ is the dimensionless parameter determining magnetic field with magnetic length $l_{B}=$ $(\hbar / e B)^{1 / 2}$, and $a^{\prime}=a / a_{0}$. Unfortunately, there is still no simple analytic solution to Eq. (14). In this case, we have to resort to the variational method to calculate the energy spectrum and the corresponding wave functions for the shallow impurity.

As the obtained laser-dressed $\widetilde{H}_{e}(\vec{R})$ is identical to the Hamiltonian of the hydrogenic system in a static magnetic field, except for the LdCP $V(R ; a)$, we can reasonably adopt similar trial wave functions for the shallow-impurity states as in Refs. [30,31], which are taken to be

$$
\psi_{n m p}(\rho, \phi, z)=\rho^{|m|} z^{p} e^{i m \phi} e^{-\xi \rho^{2}-\zeta R}\left(1-\lambda R_{\sigma}\right),
$$

where $n, m$, and $p=0,1$ are, respectively, the principal, magnetic, and conserved $z$-parity quantum numbers with $|m|<n$. Note that these wave functions have been verified to be very accurate in describing the shallow-impurity states under a static magnetic field [31]. Here, $\xi$ and $\zeta$ are two variational parameters, and $\sigma$ is an additional one for the $2 s$ state, which are determined such that they minimize the unperturbed energy

$$
E_{n m p}^{(0)}=\frac{\left\langle\psi_{n m p}\left|\widetilde{H}_{e}(\vec{R})\right| \psi_{n m p}\right\rangle}{\left\langle\psi_{n m p} \mid \psi_{n m p}\right\rangle} .
$$

In Eq. (15), $\lambda$ is nonzero only for the $2 s$ state and is chosen such that this state is orthogonal to the ground state, $R=\left(\rho^{2}+z^{2}\right)^{1 / 2}$, and $R_{\sigma}=\left(\rho^{2}+\sigma^{2} z^{2}\right)^{1 / 2}$. In this study, we only consider the following states in the calculation: $1 s=|1,0,0\rangle, 2 s=|2,0,0\rangle, 2 p_{z}=|2,0,1\rangle, 2 p^{ \pm}=|2, \pm 1,0\rangle$, $3 d^{-2}=|3,-2,0\rangle$, and $4 f^{-3}=|4,-3,0\rangle$. This is because these lowest impurity states are the most likely to be observed in experiments, which are the relevant states only needed to study the RMPE on shallow-impurity states in semiconductors in a magnetic field [30-32]. In addition, we have found that the influence of the external fields on the impurity states with different quantum numbers is similar, so that a similar behavior of the higher excited impurity states can be observed.

With the variational wave functions given as Eq. (15) and the Hamiltonian $\widetilde{H}_{e}(\vec{R})$ shown in Eq. (14), we can derive the following expressions for the diagonal matrix elements: (1) for all the shallow-impurity states apart from $2 s$ which can be 
simplified to a twofold numerical integral

$$
\begin{gathered}
\left\langle\psi_{n m p} \mid \psi_{n m p}\right\rangle=Q(|m|, p, 0) \\
\left\langle\psi_{n m p}\left|\widetilde{H}_{e}\right| \psi_{n m p}\right\rangle=\left[\gamma m+4 \xi(|m|+1)-\zeta^{2}\right] Q(|m|, p, 0)+2 \zeta(|m|+p+1) Q(|m|, p,-1)+p(1-p) Q(|m|, p-1,0) \\
+\left(\frac{\gamma^{2}}{4}-4 \xi^{2}\right) Q(|m|+1, p, 0)-4 \xi \zeta Q(|m|+1, p,-1)+W(|m|, p)
\end{gathered}
$$

where we have defined the function

$$
Q(i, j, k)=\frac{(-1)^{i} \pi}{2^{i+2 j+k}} \frac{\partial^{i}}{\partial \xi^{i}} \int_{0}^{\infty} d z e^{-2 z(\zeta+\xi z)} \frac{\partial^{2 j}}{\partial(\zeta+2 \xi z)^{2 j}} \frac{\Gamma[k+2,2 z(\zeta+2 \xi z)]}{(\zeta+2 \xi z)^{k+2}} e^{2 z(\zeta+2 \xi z)}
$$

and the functions $W(i, j)$ induced by the LdCP

$$
W(i, j)=-8 \pi \int_{0}^{\infty} d \rho \rho^{2 i+1} e^{-2 \xi \rho^{2}} \int_{0}^{\infty} d z z^{2 j} e^{-2 \zeta R} W_{0}
$$

with $W_{0}=\left[1+3\left(8 R^{2} a^{\prime 2}+a^{\prime 4} \eta_{0}^{2}\right) / 16\left(R^{2}+a^{\prime 2}\right)^{2}\right] /\left(R^{2}+a^{\prime 2}\right)^{1 / 2}$ and $\Gamma(k, z)$ being the incomplete gamma function; and (2) for the $2 s$ state which involves two different twofold numerical integrals

$$
\left\langle\psi_{2 s} \mid \psi_{2 s}\right\rangle=4 \pi \int_{0}^{\infty} d \rho \rho e^{-2 \xi \rho^{2}} \int_{0}^{\infty} d z e^{-2 \zeta R}\left(1-\lambda R_{\sigma}\right)^{2}
$$

and

$$
\begin{aligned}
\left\langle\psi_{2 s}\left|\widetilde{H}_{e}\right| \psi_{2 s}\right\rangle= & 4 \pi \int_{0}^{\infty} d \rho \rho e^{-2 \xi \rho^{2}} \int_{0}^{\infty} d z e^{-2 \zeta R}\left(1-\lambda R_{\sigma}\right) \\
& \times\left\{\left(1-\lambda R_{\sigma}\right)\left[4 \xi-\zeta^{2}+\frac{2 \zeta\left(1-2 \xi \rho^{2}\right)}{R}+\left(\frac{\gamma^{2}}{4}-4 \xi^{2}\right) \rho^{2}-2 W_{0}\right]+\frac{\lambda \sigma^{2} R^{2}}{R_{\sigma}^{3}}-\frac{\lambda\left(4 \xi \rho^{2}-1\right)}{R_{\sigma}}-\frac{2 \lambda \zeta R_{\sigma}}{R}\right\} .
\end{aligned}
$$

In addition, here we only need to calculate the energy levels of shallow-impurity states with $m \geqslant 0$ because of the exact relation $E_{-|m|}=E_{|m|}-2|m| \gamma$.

The binding energy for the $i$ th $[i=(n, m, p)]$ shallowimpurity state is defined as follows:

$$
E_{i}^{b}=(2 N+1) \gamma-E_{i}^{(0)},
$$

where $N$ is the Landau level (LL) quantum number of the system in the presence of the two external fields. For all the considered states except $2 p^{+}, N=0$ corresponds to the first LL. For the $2 p^{+}$state, one has $N=1$.

\section{Magnetopolaron correction}

With the electron wave function, in the presence of a timedependent scattering potential $V(t)=V e^{\delta t}$, one can derive the energy shift of the $i$ th state using time-dependent perturbation theory (TdPT) [48] up to the second order on the scattering potential

$$
\triangle E_{i}=V_{i i}+\sum_{j} \frac{\left|V_{i j}\right|^{2}}{E_{i}^{(0)}-E_{j}^{(0)}+i \hbar \delta} .
$$

Here, $V$ is assumed to be constant and $\delta$ is small and positive. The first and second terms on the right-hand side of Eq. (22), respectively, correspond to the first- and second-order energy shifts induced by the scattering potential $V(t)$. It should be mentioned that $\delta$ is not necessarily a real number because this energy shift can be generalized to a harmonic perturba- tion. Most importantly, the real part of this energy shift is associated with the well-known level shift obtained by using time-independent perturbation theory [48], namely,

$$
\operatorname{Re}\left(\triangle E_{i}\right)=V_{i i}+\sum_{j} \frac{\left|V_{i j}\right|^{2}}{E_{i}^{(0)}-E_{j}^{(0)}+\triangle_{i}},
$$

where we have introduced an important physical quantity, namely, $\Delta_{i}$ in the denominator of Eq. (23). On the one hand, $\triangle_{i}=0$ when $\delta$ is infinitesimally small, then the obtained level shift is equivalent to that obtained from Rayleigh-Schrödinger perturbation theory (RSPT) which has been widely applied to evaluate the magnetopolaron correction (MPC) to donor states in the nonresonant region [30,31,33-35]. On the other hand, we can reasonably define $\triangle_{i}=\operatorname{Re}\left(\Delta E_{i}\right)$ when $\delta$ is a small but finite quantity, then Eq. (23) is completely in line with the result obtained from Wigner-Brillouin perturbation theory (WBPT) which has also been extensively used to investigate the RMPE of donor states in the resonant region [30,31,33-35].

The MPC to the energy of shallow-impurity states in weak polar semiconductor systems can be reasonably characterized by second-order perturbation theory (i.e., RSPT and WBPT). As a consequence, with the LdIP for the e-p interaction obtained as Eq. (12) and the trial electron wave functions given as Eq. (15), the RMPE on shallow-impurity states can be investigated following the above TdPT in semiconductor systems under the two external fields. By applying the TdPT to our case, the MPC of the shallow-impurity energy for the 
$i$ th state is calculated by

$$
\triangle E_{i}=-\sum_{j, n^{\prime}} \sum_{\vec{Q}} \frac{\left|\left\langle\psi_{j} ; \vec{Q}\left|H_{I}\right| \psi_{i} ; \overrightarrow{0}\right\rangle\right|^{2} J_{n^{\prime}}^{2}[Z(q)]}{\hbar \omega_{\vec{Q}}+n^{\prime} \hbar \omega+E_{j}^{(0)}-E_{i}^{(0)}-\triangle_{i}}
$$

Here, $\triangle_{i}=0$ for all shallow-impurity states in the nonresonant polaron region corresponding to the RSPT, $\triangle_{2 p^{+}}=\Delta E_{2 p^{+}}-$ $\triangle E_{1 s}$ for the $2 p^{+}$state in the resonant polaron region corresponding to the improved WBPT (IWBPT) [30,31,33-35], and $\left|\psi_{j} ; \vec{Q}\right\rangle$ describes a state composed by an electron with unperturbated energy $E_{j}^{(0)}$ and a LO phonon with momentum $\hbar \vec{Q}$ and energy $\hbar \omega_{\vec{Q}}$. In the presence of an ITLF, the RMPE on shallow-impurity states in semiconductor systems can be accompanied by the emission and absorption of photons. In Eq. (24), $n^{\prime}>0, n^{\prime}<0$, and $n^{\prime}=0$ correspond, respectively, to $n$-photon absorption, $n$-photon emission, and elastic-photon scattering. In the absence of the ITLF (i.e., $F_{0} \rightarrow 0$ ), due to $\lim _{x \rightarrow 0} J_{n^{\prime}}(x)=\delta_{n^{\prime}, 0}$, one has

$$
\triangle E_{i}=-\sum_{j} \sum_{\vec{Q}} \frac{\left|\left\langle\psi_{j} ; \vec{Q}\left|H_{I}\right| \psi_{i} ; \overrightarrow{0}\right\rangle\right|^{2}}{\hbar \omega_{\vec{Q}}+E_{j}^{(0)}-E_{i}^{(0)}-\triangle_{i}},
$$

which is the well-known result obtained previously for the MPC to the energy levels of shallow-impurity states in semiconductor systems when only subjected to a static magnetic field [30,31] using the RSPT and IWBPT.

In order to calculate the MPC to the energy of the $i$ th shallow-impurity state, in principle, one has to include all shallow-impurity states in the sum $\sum_{j}$ in Eq. (24), which is a formidable task, especially for the polaron nonresonant region where the sum over the shallow-impurity states converges very slowly because no single state in this sum dominates. However, if we limit this sum to a finite number, it will result in a systematical underestimation of the MPC. Fortunately, it has been found that we only need to include a few relevant electronic states to calculate the MPC to the energy of shallow-impurity states using a method proposed previously [49,50], which has been extensively and successfully applied to study the RMPE on shallow-impurity states in semiconductor systems under magnetic field [30,31].

Following the method described in Refs. [49,50], we can evaluate the MPC approximately by formally including all shallow-impurity states in the sum $\sum_{j}$, so that Eq. (24) can be rewritten as

$$
\begin{aligned}
\triangle E_{i}= & -\alpha \hbar \omega_{\mathrm{LO}}-\sum_{j, n^{\prime}} \sum_{\vec{Q}}\left|\left\langle\psi_{j} ; \vec{Q}\left|H_{I}\right| \psi_{i} ; \overrightarrow{0}\right\rangle\right|^{2} J_{n^{\prime}}^{2}[Z(q)] \\
& \times \frac{\left(E_{i}^{(0)}+\triangle_{i}+Q^{2}-E_{j}^{(0)}-n^{\prime} \hbar \omega\right)^{2}}{\left(\hbar \omega_{\mathrm{LO}}+Q^{2}\right)^{2}\left(\hbar \omega_{\mathrm{LO}}+n^{\prime} \hbar \omega+E_{j}^{(0)}-E_{i}^{(0)}-\triangle_{i}\right)},
\end{aligned}
$$

where the LO phonon frequency has been considered to be independent of its wave vector in the long-wavelength range, say, $\omega_{\vec{Q}}=\omega_{\text {LO }}$. Here, Eq. (25) consists of two terms: the first $-\alpha \hbar \omega_{\mathrm{LO}}$ is the polaron correction of a free electron in the field-free case, and the second term is the MPC attributed to the two external applied fields and depends on the specific shallowimpurity states. As a result, the calculation of the MPC to the energy of shallow-impurity states is reduced to the evaluation of the second term on the right-hand side of Eq. (25).

In the polaron nonresonant region, a similar expression for the MPC as in Refs. [30,31] can be found,

$$
\triangle E_{i}=-\alpha\left(\hbar \omega_{\mathrm{LO}}+S_{i}\right)
$$

where $S_{i}=(2 / 3)\left\langle\psi_{i}\left|\widetilde{H}_{e}(\vec{R})-2 V(R ; a)\right| \psi_{i}\right\rangle$, which can be simplified as

$$
S_{i}=\frac{2}{3}\left(E_{i}^{(0)}+\frac{\tilde{W}\left(\left|m_{i}\right|, p_{i}\right)}{\left\langle\psi_{i} \mid \psi_{i}\right\rangle}\right) .
$$

Obviously, the photon process contributes absolutely nothing towards the MPC in the polaron nonresonant region. Here, $E_{i}^{(0)}$ is the unperturbed energy of the $i$ th state obtained by the above variational method, and the function $\widetilde{W}\left(\left|m_{i}\right|, p_{i}\right)$ is defined as

$$
\begin{aligned}
\widetilde{W}\left(\left|m_{i}\right|, p_{i}\right)= & -8 \pi \int_{0}^{\infty} d \rho \rho^{2\left|m_{i}\right|+1} e^{-2 \xi \rho^{2}} \int_{0}^{\infty} d z z^{2 p_{j}} \\
& \times e^{-2 \zeta R}\left(1-\lambda R_{\sigma}\right)^{2} W_{0}
\end{aligned}
$$

for the $i$ th shallow-impurity state with the magnetic quantum number $m_{i}$ and conserved $z$-parity quantum number $p_{i}$.

In the vicinity of the polaron resonant region, the MPC to the $2 p^{+}$state can not be evaluated using Eq. (26). Following the method used in Refs. [30,31], however, the MPC to the $2 p^{+}$state can be calculated as

$$
\begin{aligned}
\triangle E_{2 p^{+}}= & \triangle E_{2 p^{-}}-\sum_{i=2 p^{ \pm}} \sum_{j, n^{\prime}} \sum_{\vec{Q}} m_{i}\left|\left\langle\psi_{j} ; \vec{Q}\left|H_{I}\right| \psi_{i} ; \overrightarrow{0}\right\rangle\right|^{2} \\
& \times \frac{\left(E_{j}^{(0)}+n^{\prime} \hbar \omega-E_{i}^{(0)}-Q^{2}\right)^{2} J_{n^{\prime}}^{2}[Z(q)]}{\left(\hbar \omega_{\vec{Q}}+Q^{2}\right)^{2}\left(\hbar \omega_{\vec{Q}}+n^{\prime} \hbar \omega+E_{j}^{(0)}-E_{i}^{(0)}-\triangle_{i}\right)},
\end{aligned}
$$

where $\triangle E_{2 p^{-}}$is the nonresonant MPC and is obtained from Eq. (26), $m_{i}$ is the magnetic quantum number of the $i$ th shallow-impurity state, and the sum $\sum_{j}$ is limited only to the relevant shallow-impurity states. In contrast to Eq. (26), the photon process plays an important role in determining the MPC to the $2 p^{+}$state in this resonant region.

Finally, notice that the band nonparabolicity plays an important role in the energy levels associated to the shallowimpurity states in GaAs bulk semiconductors for the case of high magnetic fields. In order to obtain the energies of the nonparabolic conduction band $E_{n p}$ from that of a parabolic conduction band $E_{p}$, we use the standard Kane model [51]

$$
E_{n p}=\frac{E_{g}}{2}\left[-1+\left(1+4 \frac{E_{p}}{E_{g}}\right)^{1 / 2}\right] \text {. }
$$

Note that this model has been proven to be successful in describing the band nonparabolicity in GaAs bulk semiconductor [52] and has been widely used in studying the effects of band nonparabolicity on the MPC for shallow-impurity states [31].

\section{RESULTS AND DISCUSSIONS}

The numerical results of this work pertain to the shallow impurity in GaAs bulk semiconductors. In the numerical calculations, we take the effective-electron mass $m^{*}=0.067 \times m_{e}$ 


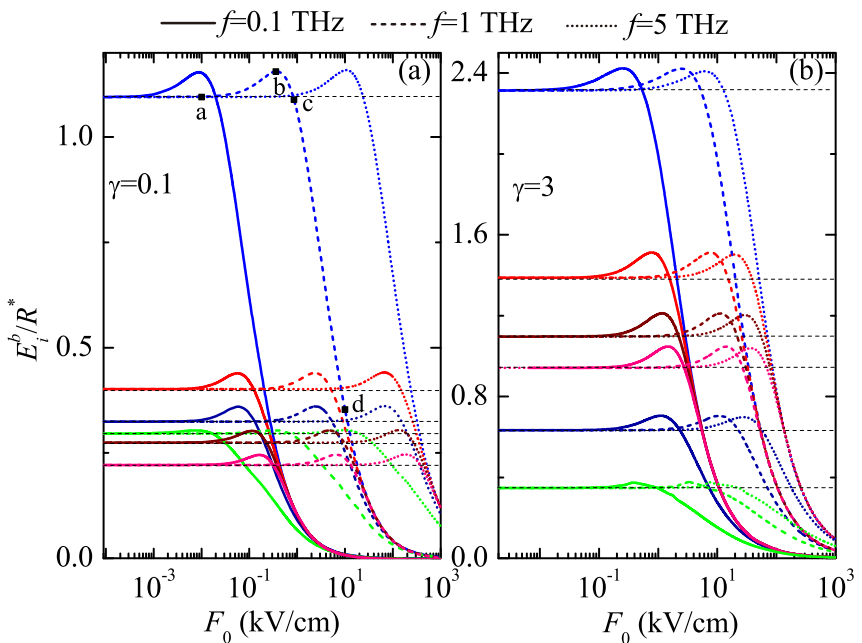

FIG. 2. The dependence of the binding energy (in units of $R^{*}$ ) for the ground $1 s$ (blue curve) and excited $2 s$ (green curve), $2 p^{ \pm}$ (red cure), $2 p_{z}$ (dark blue curve), $3 d^{-2}$ (dark red curve), and $4 f^{-3}$ (pink curve) states as a function of the laser-field intensity at fixed magnetic fields $\gamma=0.1$ (left figure) and $\gamma=3.0$ (right figure) for three different laser field frequencies: $0.1 \mathrm{THz}$ (solid curve), $1 \mathrm{THz}$ (dashed curve), and $5 \mathrm{THz}$ (dotted curve). Here, the horizontal dashed lines represent the shallow-impurity binding energies in the absence of the ITLFs.

with $m_{e}$ being the free-electron mass, the high-frequency dielectric constant $\varepsilon=10.9$ when irradiated by ITLFs, and the band gap $E_{g}=1520 \mathrm{meV}$.

In order to check the validity of our results, first we have calculated the energy levels of a shallow impurity in GaAs semiconductors only under a static magnetic field with or without the electron-phonon interaction. The comparison shows that our results are consistent with those of Ref. [30]. This section is organized as follows. In Secs. III A and III B we respectively outline the ITLF and magnetic field effects on the binding energy of the shallow-impurity states and discuss the related physical reasons in detail. The MPC to the shallow-impurity states with photon process is discussed in Sec. IIIC and the RMPE accompanied by the emission and absorption of photons is calculated in Sec. IIID.

\section{A. ITLF effect on the binding energy of the shallow-impurity states}

The influence of ITLFs on the binding energy of the lowest states, namely, $1 s, 2 s, 2 p^{ \pm}, 2 p_{z}, 3 d^{-2}$, and $4 f^{-3}$, in GaAs semiconductors at fixed magnetic field is shown in Figs. 2 and 3. As a reference we have also plotted the binding energy of the shallow-impurity states under a static magnetic field indicated by horizontal dashed lines in these figures. Note that the LdCP is obviously affected by the electromagnetic fields through the field parameter $a=\left|r_{0}\right|\left[\left(1+\eta^{2}\right) / 2\right]^{1 / 2} \propto F_{0} /\left|\omega^{2}-\omega_{c}^{2}\right|$ as reflected in Eq. (11), so that the laser field intensity and frequency affect the binding energy in an opposite way in the region away from $\omega_{c}$.

From Fig. 2 it is clear that, in the low laser field intensity limit, the binding energy is not affected by $F_{0}$ and remains the static one as calculated in Ref. [30]. As the laser field
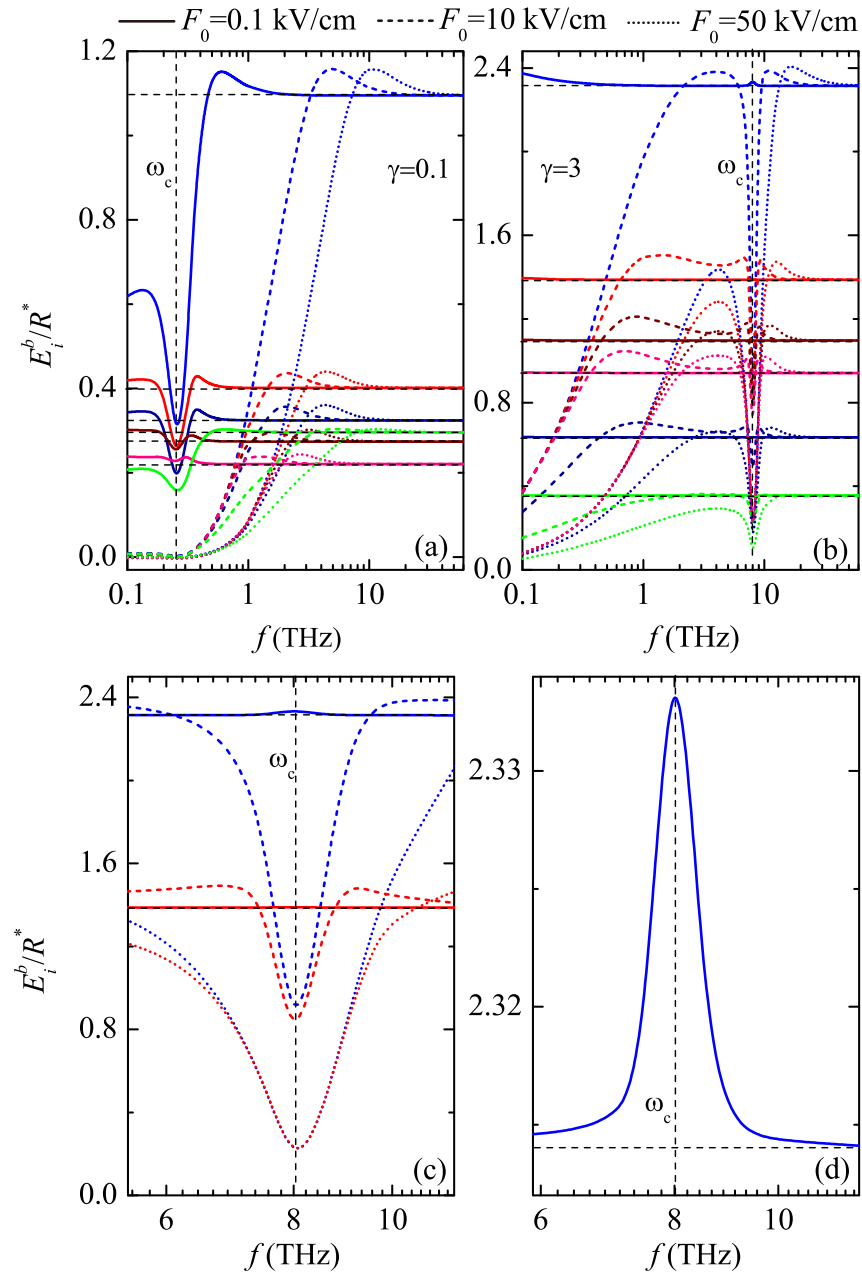

FIG. 3. The dependence of the binding energy for the ground $1 s$ (blue curve) and excited $2 s$ (green curve), $2 p^{ \pm}$(red cure), $2 p_{z}$ (dark blue curve), $3 d^{-2}$ (dark red curve), and $4 f^{-3}$ (pink curve) states as a function of the laser field frequency at fixed magnetic field for different laser field intensities as indicated. In (c) and (d) the vicinity of the cyclotron frequency of (b) is enlarged, the dashed horizontal lines depict the binding energy for the laser-field-free case, and $\omega_{c}$ is shown to indicate the position of the cyclotron frequency.

intensity increases, the shallow-impurity binding energy increases slowly at first, and after it reaches its maximum, which shifts to higher laser field intensity with an increase of laser field frequency, the binding energy rapidly decreases with increasing $F_{0}$, tending to approach each other, and finally it decreases to zero in the high laser field intensity limit. This implies that the shallow impurity has been ionized, which is opposite to previous results in the absence of magnetic field [13]. In addition, this nonmonotonic dependence of the binding energy on $F_{0}$ is also very different from previous monotonic behavior of the binding energy only under ITLFs [13-16].

In the region away from $\omega_{c}$, in contrast to Fig. 2, as expected an opposite nonmonotonic tendency of the binding energy as function of $f$ is observed in Figs. 3(a) and 3(b). In the vicinity of $\omega_{c}$, more interestingly, Fig. 3 shows an appreciable effect of cyclotron motion on the binding energy. In this way, a very pronounced dip appears exactly at $\omega_{c}$ and becomes more and more significant with increasing $F_{0}$ except for a special case: 

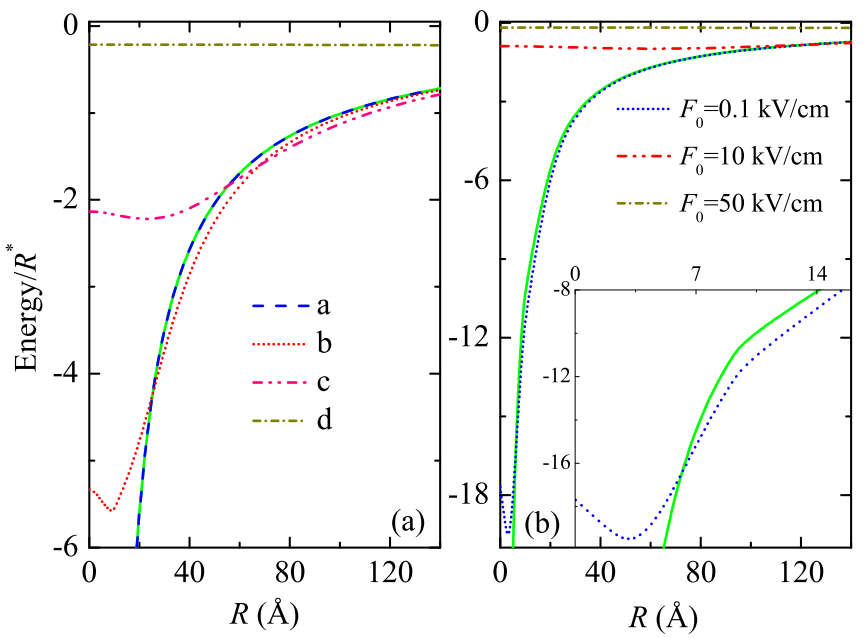

FIG. 4. Comparison of the bare Coulomb potential (green solid curve) with the LdCP for a shallow impurity in semiconductors at fixed magnetic field $\gamma=0.1$ (left figure) and $\gamma=3$ (right figure) for different laser field intensities when (a) $f=1 \mathrm{THz}$ and (b) $f=$ $8 \mathrm{THz}$. In (a) $a, b, c$, and $d$ correspond to the points indicated in Fig. 2(a) and in (b) the radiation frequency is in the vicinity of $\omega_{c}$, corresponding to Fig. 3(b). Inset: the region with small $R$ is enlarged.

$\gamma=3$ and $F_{0}=0.1 \mathrm{kV} / \mathrm{cm}$ as seen in Fig. 3(d). In this peculiar case, on the contrary, the binding energy of the ground state exhibits an appreciable peak at $\omega_{c}$. It should be pointed out that such intriguing behavior of the binding energy seen in Fig. 3(d) is also observed for the other impurity states. Moreover, these tendencies of the binding energy with $f$ can only be observed in the Faraday configuration.

For a better understanding of these behaviors of the binding energy associated with the shallow-impurity states, we have plotted the LdCP at fixed magnetic field and laser field frequency for increasing laser field intensity in Fig. 4. In Fig. 4(a), $a$ (blue dashed curve), $b$ (red dotted curve), $c$ (pink dashed-dotted-dotted curve), and $d$ (dark yellow dashed-dotted curve) correspond to points shown in Fig. 2(a), which are far away from the cyclotron frequency. From Fig. 4(a), it is clear that the laser field intensity effect on the LdCP can be reasonably neglected when $F_{0}$ is less than or equal to that at point $a$ in Fig. 2(a) with the same magnetic field and radiation frequency. When $F_{0}$ changes from $a$ to $d$, the LdCP is strongly influenced by the $F_{0}$ in the short-range interaction strength while the long-range interaction strength is unaffected. Let us see in more detail what happens in this short-range interaction strength region. When $F_{0}$ changes from $a$ to $b$, the $\mathrm{LdCP}$ decreases in the region with small $R$ and increases in the region with large value of $R$, on average, the average LdCP is enhanced and reaches its maximum at point $b$. This is evident by the fact that the binding energy starts with the static binding energy and grows to a maximum when $F_{0}$ increases from $a$ to $b$, as shown in Fig. 2(a). After that the average LdCP decreases with increasing $F_{0}$ and becomes equivalent to the effect of the bare Coulomb potential at point $c$, and then it rapidly decreases. This is the main reason why the binding energy decreases with increasing $F_{0}$ when it varies from $b$ to $d$, as shown in Fig. 2.

When the radiation field is intense enough [for instance, when $F_{0}$ is larger than that at point $d$ in Fig. 2(a) for the same
$B$ and $f$ ], the average LdCP becomes weak enough, so that the magnetic force becomes much more effective in binding the electrons in the plane perpendicular to the magnetic field. In this way, the bound $1 s, 2 s, 2 p_{z}, 2 p^{-}, 3 d^{-2}$, and $4 f^{-3}$ states are then just lying below the first LL of a free electron where the energy levels of different impurity states begin to approach each other and finally decrease to zero, as shown in Fig. 2. By considering the definition of the binding energy given in Eq. (21) and the relation $E_{-|m|}=E_{|m|}-2|m| \gamma$, the binding energies for the $2 p^{-}$and $2 p^{+}$states are equal to each other as seen in Fig. 2. Moreover, due to the opposite effect of $F_{0}$ and $f$ on the LdCP in the region away from $\omega_{c}$, the peak of the binding energy shifts towards higher $F_{0}$ with increasing $f$ as displayed in Fig. 2, which is also the physical reason why the binding energy exhibits a nonmonotonic function of $f$ in Figs. 3(a) and 3(b).

In the vicinity of $\omega_{c}$, the variation of the LdCP with $F_{0}$ is displayed in Fig. 4(b) at fixed $\gamma=3$ and $f=8 \mathrm{THz}$, corresponding to the case in the vicinity of $\omega_{c}$ as shown in Fig. 3(b). In this case, obviously, the LdCP can also be slightly enhanced so long as $F_{0}$ is weak enough while it remarkably decreases in high $F_{0}$. This explains two distinct opposite tendencies of the binding energy with $f$ as shown in Fig. 3, namely, a pronounced peak and an appreciable dip are simultaneously observed at $\omega_{c}$ as seen in Figs. 3(a)-3(c) and $3(\mathrm{~d})$, respectively.

To provide further insights into these behaviors of the binding energy observed in Figs. 2 and 3, the effective widths of the wave functions associated with the different shallow-impurity states are calculated. Within the Ehlotzky approach [7], the effective widths in the $x-y$ plane and in the $z$ direction can be calculated as $r_{\rho}=\left[\left\langle\rho^{2} / 2\right\rangle+a^{2} / 2\right]^{1 / 2}$ and $r_{z}=\left[\left\langle z^{2}\right\rangle\right]^{1 / 2}$, respectively. We find that $r_{\rho}$ is remarkably affected by the electromagnetic field via the field parameter $a$. The numerical results (in units of $a_{0}$ ) for the ground state as a function of laser field intensity and frequency at fixed magnetic field are displayed in Figs. 5 and 6, respectively. Here, we do not plot the widths associated with the other states since they have similar qualitative dependence on the ITLFs as the ground state.

From Figs. 5 and 6 it is clear that the ground state is unaffected by the ITLFs in the low- $F_{0}$ limit or in the high- $f$ limit, i.e., the widths $r_{\rho}$ and $r_{z}$ remain the static widths. In contrast, in the opposite limit of $F_{0}$ and $f$, both $r_{\rho}$ and $r_{z}$ for the $1 s$ state exponentially increase and, correspondingly, the electron wave function is spread out extensively, which results in an ionization of the shallow impurity (i.e., the binding energy approaches zero) as shown in Figs. 2 and 3. Meanwhile, $r_{\rho}$ is much larger than $r_{z}$ because the ITLF is polarized in the $x-y$ plane, which has a direct effect on $r_{\rho}$ but an indirect effect on $r_{z}$, resulting in a worse localization in this plane. As we can see clearly from Figs. 5(e)-5(h), both $r_{\rho}$ and $r_{z}$ decrease appreciably at first and after they reach their minimum, which shifts towards higher $F_{0}$ with increasing $f, r_{\rho}$, and $r_{z}$ increase rapidly with increasing $F_{0}$. In other words, with increasing $F_{0}$ the ground state first becomes more localized, but after a critical value of $F_{0}$, the ground state is spread out rapidly, which results in a similar variation of the binding energy with $F_{0}$ as shown in Fig. 2. Moreover, an opposite trend of the localization of the ground state with $f$ is also observed in Figs. 6(e)-6(h) in the region away from $\omega_{c}$, 

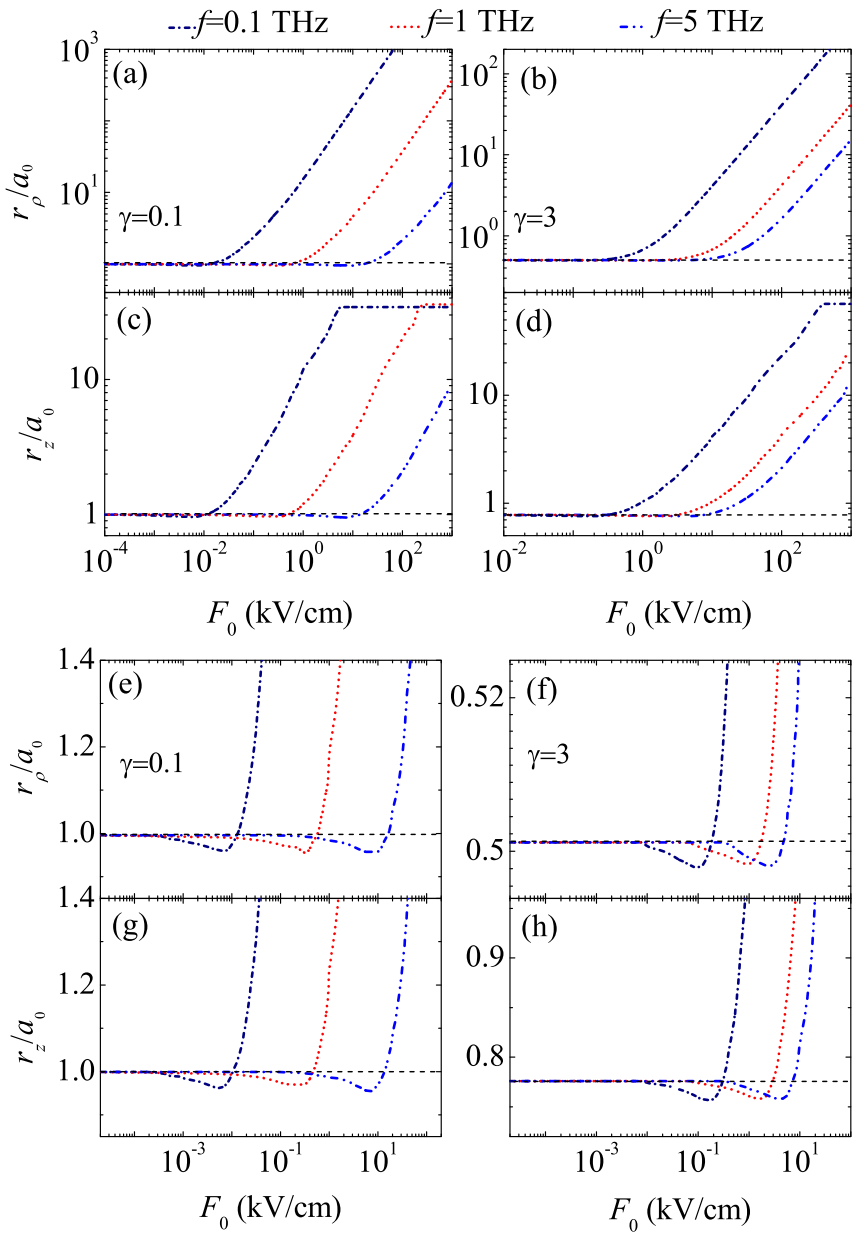

FIG. 5. The widths of the wave function for the $1 s$ ground state in the $x-y$ plane and in the $z$ direction in units of $a_{0}$ as function of the laser field intensity at fixed magnetic field for different laser field frequencies as indicated. In (e)-(h) the small width regions of (a)-(d) are enlarged, respectively. The dashed horizontal lines depict the widths associated with the considered impurity states in the absence of the ITLFs.

which is the reason why we have a nonmonotonic trend of the binding energy with $f$ in Figs. 3(a) and 3(b).

In the vicinity of $\omega_{c}$, more interestingly, both $r_{\rho}$ and $r_{z}$ exhibit a peak at $\omega_{c}$ except for the case when $\gamma=3$ and $F_{0}=0.1 \mathrm{kV} / \mathrm{cm}$ where a pronounced dip appears at $\omega_{c}$ as shown in Figs. 6(f) and 6(h). That is to say, the ground state is more spread out at $\omega_{c}$ except for that particular case where the electron wave function becomes more localized, leading to two distinct opposite behaviors of the binding energy in Fig. 3. Moreover, $r_{z}$ exhibits a plateau in the high- $F_{0}$ limit in Figs. 5(c) and 5(d), which is due to the fact that the shallow impurity exhibits a Landau-like state [53] due to the weakness of the LdCP and $F_{0}$ has no direct effect on $r_{z}$.

By comparing Figs. 2-6, one can see that the dependence of the binding energy on the ITLFs is physically consistent with the variation of the LdCP and the widths $r_{\rho}$ and $r_{z}$ (i.e., the localization of the shallow-impurity states) with the ITLFs, namely, these behaviors of the binding energy can be reasonably understood in terms of the electron-localization properties in bulk semiconductors which is effectively adjusted
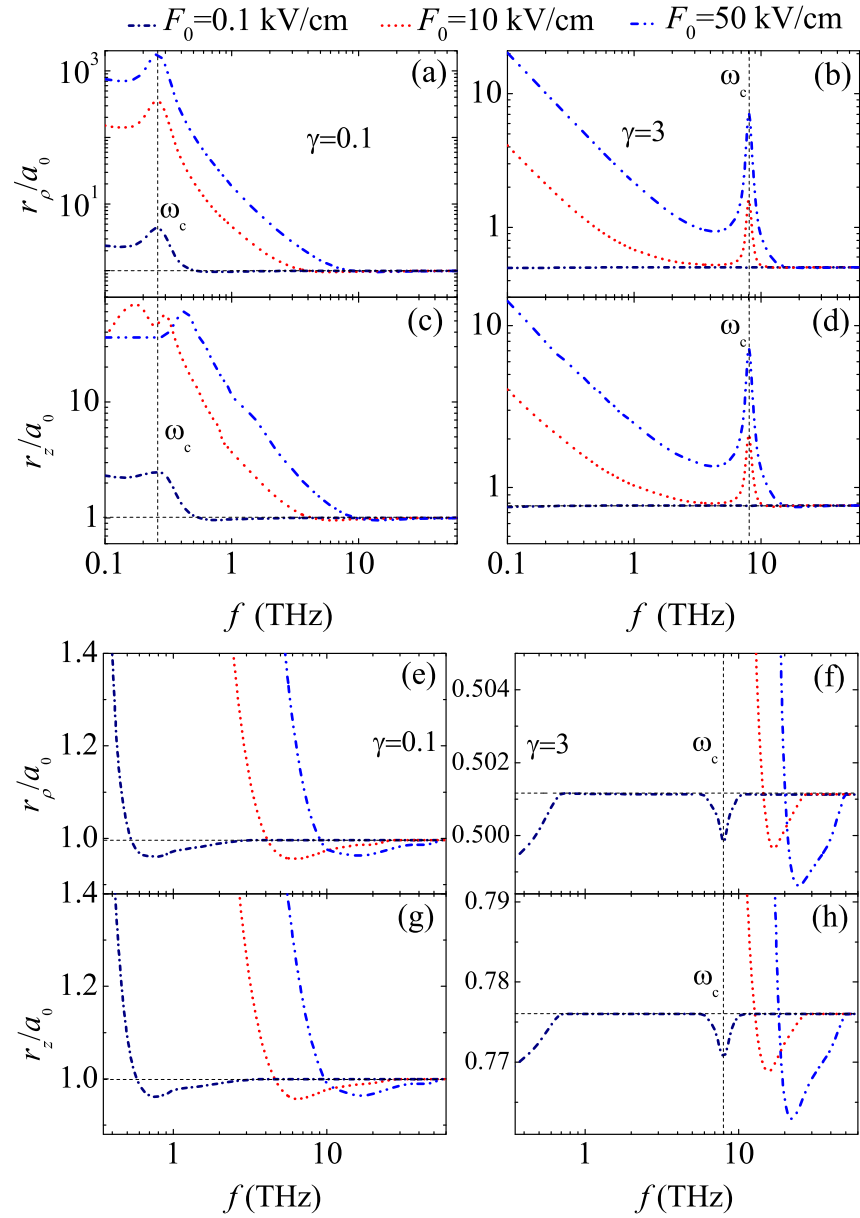

FIG. 6. The widths of the wave function for the ground state in the $x-y$ plane and in the $z$ direction in units of $a_{0}$ and as function of the laser field frequency at fixed magnetic field for different laser field intensities as indicated. In (e)-(h) the small width regions of (a)-(d) are enlarged. $\omega_{c}$ is shown to indicate the position of the cyclotron frequency, and the dashed horizontal lines indicate the widths associated with the considered impurity states in the absence of the ITLFs.

by the ITLFs through the LdCP. Increasing the LdCP leads to a decrease of the widths $r_{\rho}$ and $r_{z}$ and, correspondingly, to an increase of the impurity-electron localization and, therefore, to an increase of the binding energy.

\section{B. Magnetic field effect on the binding energy of the shallow-impurity states}

The binding energy associated with shallow-impurity states is displayed in Fig. 7 as a function of the magnetic field and for several values of the laser field intensity at fixed laser field frequency. It should be pointed out that, when $F_{0}$ is low enough (i.e., $F_{0}=10^{-5} \mathrm{kV} / \mathrm{cm}$ for $f=0.1 \mathrm{THz}$ and $F_{0}=10^{-3} \mathrm{kV} / \mathrm{cm}$ for $f=1 \mathrm{THz}$ ), the binding energy is equal to the static one since the effects of the ITLFs can be neglected in this case. Note that the applied magnetic field not only influences the LdCP via the field parameter $a=$ $\left|r_{0}\right|\left[\left(1+\eta^{2}\right) / 2\right]^{1 / 2} \propto F_{0} /\left|\omega^{2}-\omega_{c}^{2}\right|$, which is similar to the effect of the laser field frequency on the $\mathrm{LdCP}$, but also results 

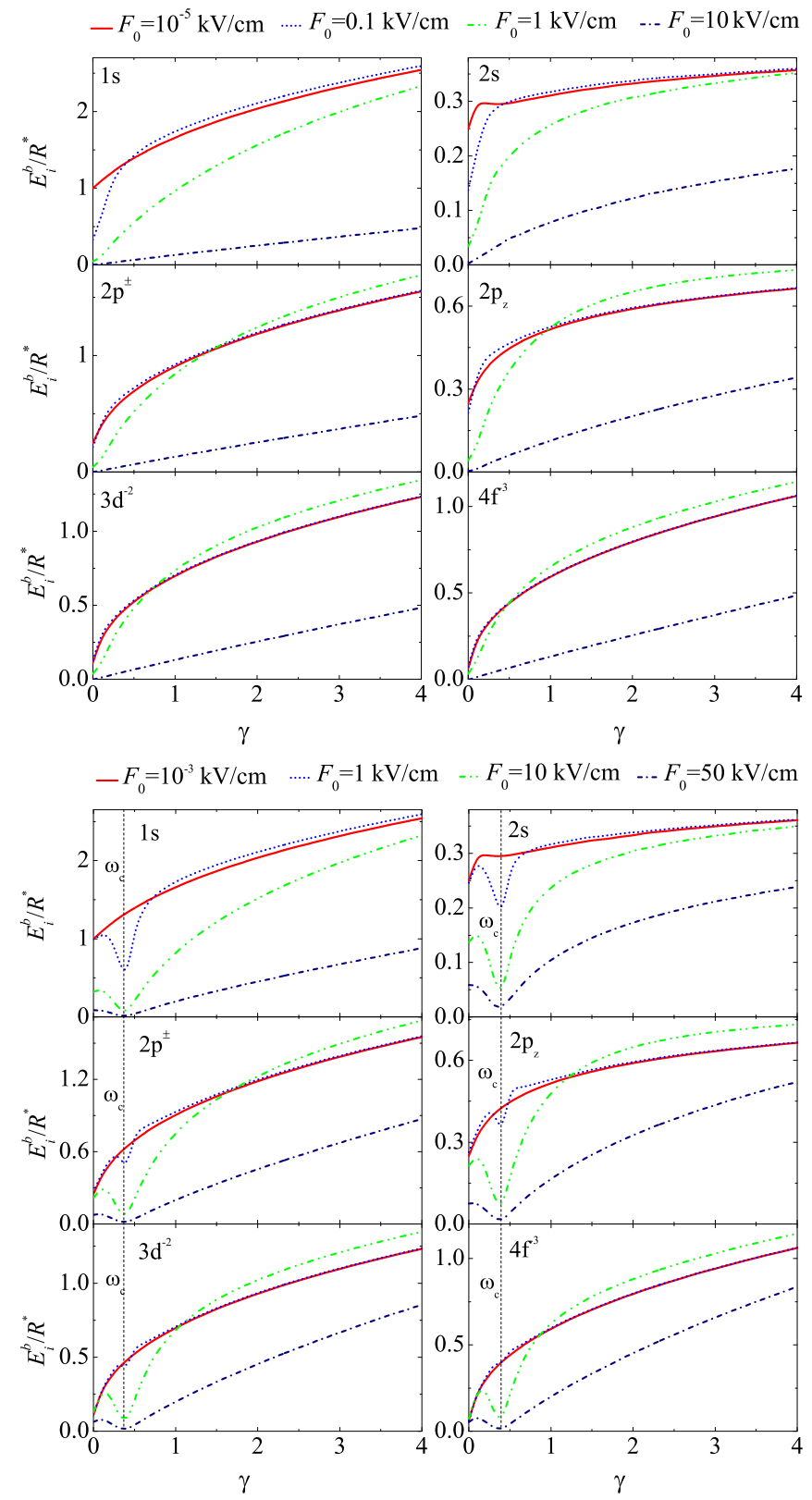

FIG. 7. The variation of the binding energy for the $1 s$ ground and excited $2 s, 2 p^{ \pm}, 2 p_{z}, 3 d^{-2}$, and $4 f^{-3}$ states in GaAs semiconductors as a function of the magnetic field at fixed $f=0.1 \mathrm{THz}$ (top figure) and $f=1 \mathrm{THz}$ (bottom figure) for different laser field intensities as indicated. Here, $\omega_{c}$ indicates the position of the cyclotron frequency.

in an additional lateral electron confinement, as reflected in Eq. (14). Consequently, the influence of the magnetic field on the binding energy is more complicated. Therefore, the widths $r_{\rho}$ and $r_{z}$ for the $1 s$ and $2 p_{z}$ states are displayed in Fig. 8 as a function of the magnetic field for several values of $F_{0}$ at fixed $f$. Here, we also do not plot the widths for the other shallow-impurity states since they have similar behaviors as these two typical states as seen in Fig. 7.

From Fig. 7 it is apparent that the binding energy is an increasing function of the magnetic field both in the absence and the presence of the ITLFs, except in the vicinity of $\omega_{c}$ where the binding energy exhibits a appreciable dip at $\omega_{c}$
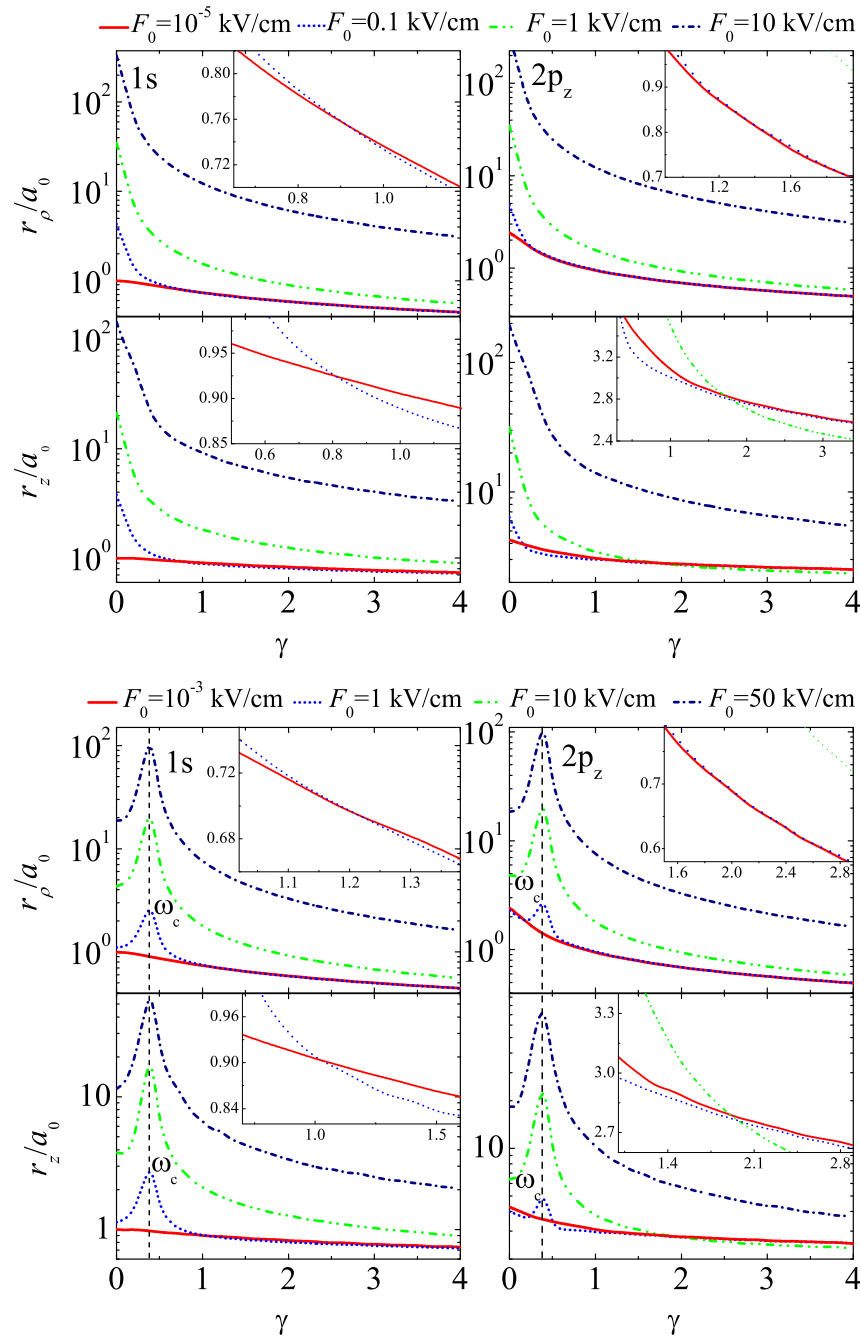

FIG. 8. The widths of the wave function for the $1 s$ ground and $2 p_{z}$ excited states in the $x-y$ plane and in the $z$ direction in units of $a_{0}$ as function of the magnetic field at fixed $f=0.1 \mathrm{THz}$ (top figure) and $f=1 \mathrm{THz}$ (bottom figure) for different laser field intensities as indicated. The small width region is enlarged in the insets and $\omega_{c}$ indicates the position of the cyclotron frequency.

and its amplitude increases with increasing $F_{0}$. The reason is that both the additional lateral confinement and the $\mathrm{LdCP}$ remarkably increase with increasing magnetic field when it moves away from $\omega_{c}$, but they rapidly decrease when the magnetic field moves towards $\omega_{c}$ or with further increase of $F_{0}$, leading to a nonmonotonic variation of the widths $r_{\rho}$ and $r_{z}$ with $f$ as seen in Fig. 8 and, therefore, to a behavior of the binding energy as shown in Fig. 7.

From the bottom of Fig. 7, at relatively low radiation levels, that is, when $F_{0} \leqslant 1 \mathrm{kV} / \mathrm{cm}$, the binding energy is smaller than the static binding energy in the region with small $\gamma$ and becomes larger than the static one in the region with large value of $\gamma$. When the radiation field is intense enough (i.e., $F_{0} \geqslant 50 \mathrm{kV} / \mathrm{cm}$ ), the binding energy is much smaller than the static binding energy. Interestingly, at the intermediate radiation levels (i.e., when $1 \mathrm{kV} / \mathrm{cm}<F_{0}<50 \mathrm{kV} / \mathrm{cm}$ ), the binding energy for the $1 s$ and $2 s$ states is smaller than the static one in the whole range of $\gamma$ while that for the $2 p_{z}, 2 p^{ \pm}$, $3 d^{-2}$, and $4 f^{-3}$ states is smaller in the region with small $\gamma$ 


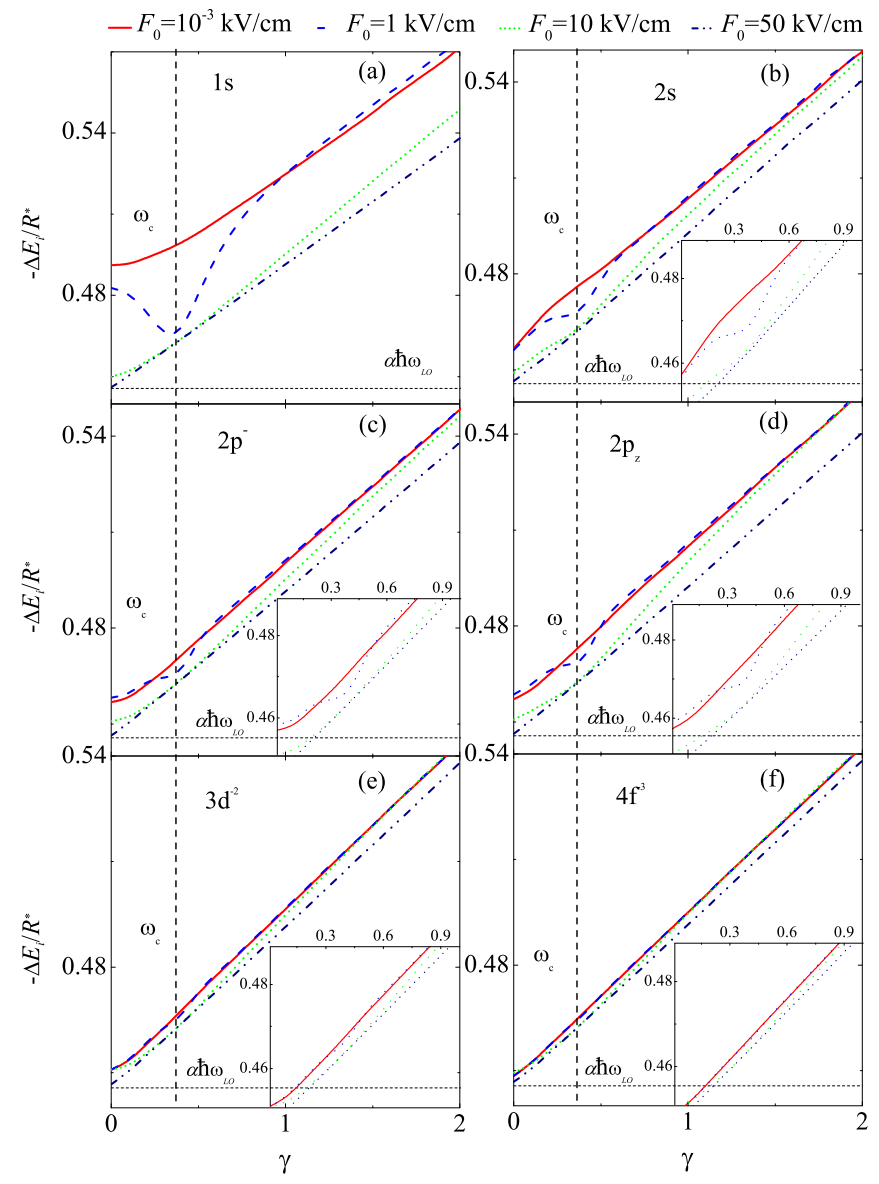

FIG. 9. Shifts of the energy levels due to the electron LO phonon interaction vs the magnetic field for a shallow impurity in GaAs semiconductors at fixed $f=1 \mathrm{THz}$ for different laser field intensities as indicated. Here, $\omega_{c}$ and $\alpha \hbar \omega_{\mathrm{LO}}$ indicate the position of the cyclotron frequency and the polaron shift of a free electron in the field-free case, respectively. Insets: the regions with small $\gamma$ are enlarged.

and becomes larger in the region with large value of $\gamma$ with respect to the static binding energy. In contrast to the bottom of Fig. 8, these behaviors of the binding energy can be reasonably explained in terms of the electron-localization properties in our systems since the change of the binding energy with magnetic field is also physically consistent with the variation of the widths with $B$. In addition, a similar behavior of the binding energy is also observed in the top of Fig. 7.

\section{Magnetopolaron correction}

In order to investigate the dependence of the MPC on the magnetic field, we have used Eq. (27) for the $2 p^{+}$state and Eq. (26) for the other states. For simplicity, we only consider the case of $f=1 \mathrm{THz}$ with single-photon process as a representative example.

The MPC to the $1 s, 2 s, 2 p^{-}, 2 p_{z}, 3 d^{-2}$, and $4 f^{-3}$ states in low magnetic fields are depicted in Fig. 9 as a function of the magnetic field for several values of laser field intensity when $f=1 \mathrm{THz}$, where the polaron shift of a free electron in the field-free case, namely, $\alpha \hbar \omega_{\mathrm{LO}}$ as reflected in Eq. (25), is indicated for reference. As a general feature, $\left|\Delta E_{i}\right| \geqslant \alpha \hbar \omega_{\mathrm{LO}}$ which can be easily proven from Eq. (25). This is due to the fact that the localization of the wave functions is greatly

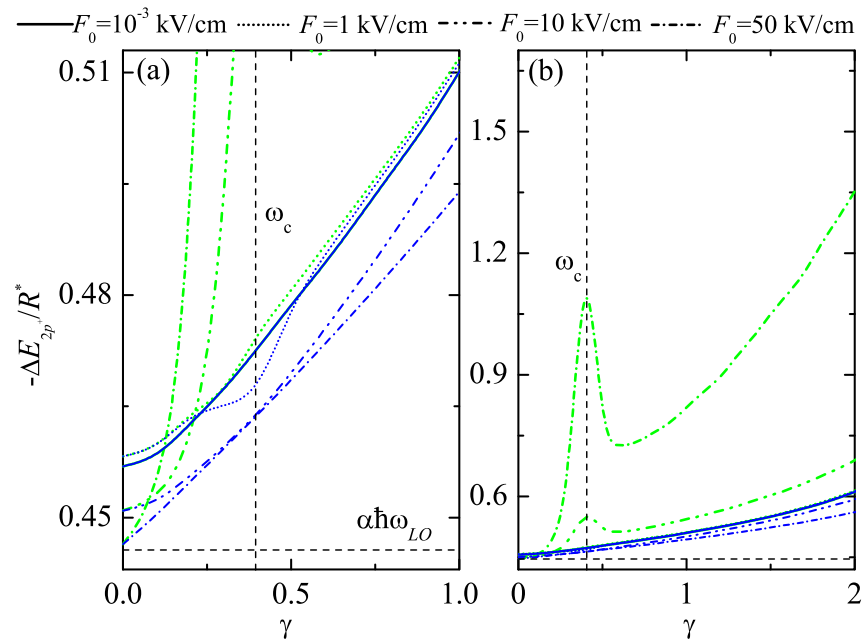

FIG. 10. Shifts of the energy levels associated with the $2 p^{+}$state with (green curve) and without (blue curve) single-photon process vs the magnetic field for a shallow impurity in GaAs at fixed $f=1 \mathrm{THz}$ for different laser field intensities as indicated. Here, $\omega_{c}$ and $\alpha \hbar \omega_{\mathrm{LO}}$ are shown to indicate the position of the cyclotron frequency and the polaron shift of a free electron in the field-free case, respectively.

enhanced by the two external applied fields, resulting in a larger MPC. Significantly, when $F_{0}=10^{-3} \mathrm{kV} / \mathrm{cm}$ the MPC is equal to the static one obtained in the absence of ITLF, which indicates that the effects of the ITLFs on the MPC can be neglected in this case.

From Fig. 9, it is clear that the MPC is an increasing function of the magnetic field in the region away from $\omega_{c}$. This is due to the fact that both the localization of the impurity states and the interaction strength of the LdIP for e-p coupling increase with increasing magnetic field when it moves away from $\omega_{c}$. On the contrary, they rapidly decrease when the magnetic field moves towards $\omega_{c}$, so that a pronounced dip is observed at $\omega_{c}$ in Fig. 9. However, this dip gradually disappears with increasing $F_{0}$. The reason is that at relatively high radiation levels, namely, when $F_{0}>1 \mathrm{kV} / \mathrm{cm}$, the difference of the binding energy between different laser field intensities at $\omega_{c}$ decreases with increasing $F_{0}$ as shown in the bottom of Fig. 7. Meanwhile, the laser field intensity plays a more and more important role in determining the LdIP for e-p interaction with increasing $F_{0}$, so that the IdIP is insensitive to the magnetic field in the high- $F_{0}$ limit, leading to the same LdIP seen from the electron in this laser-driven system. In this way, the dip gradually decreases with $F_{0}$ and disappears when $F_{0}=50 \mathrm{kV} / \mathrm{cm}$ as seen in Fig. 9 .

Figure 9 also shows that the MPC can be effectively adjusted by the laser field intensity to be red-and blue-shifted with respect to the static MPC, which is similar to the behaviors of the binding energy as shown in the bottom of Fig. 7 . However, the quantity of the red- and blue-shift of the MPC is much smaller than that of the binding energy, which is due to the fact that the interaction strength of the LdIP for e-p coupling decreases with increasing $F_{0}$. In addition, the influences of $F_{0}$ on the MPC for the $3 d^{-2}$ and $4 f^{-3}$ states can be almost neglected when $F_{0} \leqslant 10 \mathrm{kV} / \mathrm{cm}$ as seen in Figs. 9(e) and 9(f).

Figure 10 shows the explicit dependence of the MPC for the $2 p^{+}$state with (green curve) and without (blue curve) the 
single-photon process on the magnetic field for several values of the laser field intensity when $f=1 \mathrm{THz}$. From Fig. 10(a) it is apparent that the MPC for the $2 p^{+}$state without photon process exhibits a behavior similar to that of the other states as shown in Fig. 9, which rapidly increases with the magnetic field for $\gamma>1.5$ since the $2 p^{+}$state approaches a resonance in this case [30].

Moreover, the MPC for the $2 p^{+}$state with single-photon process is equal to that without photon process when $F_{0}=$ $10^{-3} \mathrm{kV} / \mathrm{cm}$, which implies that the effects of the photon process on the MPC for the $2 p^{+}$state can be reasonably neglected at low $F_{0}$. With further increase of $F_{0}$, the MPC, including the effects of the single-photon process, becomes larger than that without photon process with increasing magnetic field since a new channel for the MPC has been opened up by taking into account the single-photon process.

More interestingly, a pronounced peak appears at $\omega_{c}$ and becomes more and more significant with increasing $F_{0}$, especially when $F_{0} \geqslant 10 \mathrm{kV} / \mathrm{cm}$ as shown in Fig. 10(b). Because in this case the binding energies for the different impurity states begin to approach each other in the vicinity of $\omega_{c}$ as shown in the bottom of Fig. 7, so that the polaron resonant region shifts towards the lower magnetic field by considering the effects of the photon process as shown in Fig. 10(b). In this way, the new opened channels play a more predominant role in determining the MPC for the $2 p^{+}$state in the vicinity of $\omega_{c}$ with increasing $F_{0}$, as reflected in Eq. (27). This explains why we have a peculiar behavior of the MPC for the $2 p^{+}$state in the vicinity of $\omega_{c}$, which in turn suggests that the action of the photon process on the MPC of the $2 p^{+}$state could be detected in this case.

\section{Resonant magnetopolaron effect}

The calculated results for the transition energy of $1 s \rightarrow 2 s$, $2 p^{ \pm}, 2 p_{z}, 3 d^{-2}$, and $4 f^{-3}$ including the effects of the polaron, single-photon process, and band nonparabolicity (solid curve) are displayed in Figs. 11-13 as a function of the magnetic field at fixed $f=1 \mathrm{THz}$ for different laser field intensities, in which the transition energy only with single-photon process is indicated by the thin dashed lines as a reference. For comparison, the results for the transition energies with the effects of polaron and band nonparabolicity (short dotteddashed curve) and with the effects of polaron and single-photon process (short dotted curve) are also presented in these figures, respectively. In Figs. $11-13, \hbar \Omega^{ \pm}=\hbar \omega_{\mathrm{LO}} \pm \hbar \omega$ corresponds to the RMPE accompanied by the absorption (region III) and emission (region I) of photons and region II corresponds to the RMPE accompanied by elastic-photon scattering. For differentiation, moreover, different branches of the $1 s \rightarrow 2 p^{+}$ transition are marked with different colors in the same region.

From Fig. 11(a) it is clear that, without considering the effects of single-photon process, near resonance only five branches of the $1 s \rightarrow 2 p^{+}$transition are observed which are a consequence of the lifting of the $E_{2 p^{+}}^{(0)}$ and $E_{i}^{(0)}+\hbar \omega_{\mathrm{LO}}$ ( $i=1 s, 2 p^{-}, 3 d^{-2}, 4 f^{-3}, 2 p_{z}$ ) degeneracies, which is similar to previous results [30]. In contrast, by including the effects of single-photon process near resonance, 10 additional branches of the $1 s \rightarrow 2 p^{+}$transition are observed which descend from the lifting of the $E_{2 p^{+}}^{(0)}$ and $E_{i}^{(0)}+\hbar \Omega^{ \pm}\left(i=1 s, 2 p^{-}\right.$, $\left.3 d^{-2}, 4 f^{-3}, 2 p_{z}\right)$ degeneracies, leading to an appreciable change of the energy levels of the shallow-impurity states in the resonant region as shown in Fig. 11(b). Moreover, the additional branches of the $1 s \rightarrow 2 p^{+}$transition can be tuned to be far away from the reststrahlen band by changing the radiation frequency.

By comparing Figs. 12 and 13 with Fig. 11, it is apparent that with increasing $F_{0}$ similar behaviors for the $1 s \rightarrow 2 p^{+}$ transition are observed in the resonant region and the characteristic anticrossing behaviors for the additional $1 s \rightarrow 2 p^{+}$ transition becomes more and more significant when $F_{0} \leqslant$ $10 \mathrm{kV} / \mathrm{cm}$, especially for the branch $E_{1 s}^{(0)}+\hbar \Omega^{-}$where an appreciable anticrossing gap is expected. Meanwhile, the energy levels of the shallow-impurity states change appreciably near resonance, which is due to the fact that not only the spectra of the shallow-impurity states, but also the LdIP for e-p coupling are strongly influenced by the ITLFs. However, Fig. 13 shows that an ambiguous anticrossing behavior for the $1 s \rightarrow 2 p^{+}$transition can be observed in the high- $F_{0}$ limit since the branches of the $1 s \rightarrow 2 p^{+}$transition are too close to be distinguished as shown in the inset of Fig. 13. This indicates that the laser field intensity and frequency may be properly chosen to generate the desired results concerning the RMPE accompanied by emission and absorption of photons in semiconductor systems. Consequently, these phenomena can be observed experimentally and in turn provide a tool to measure the e-p interaction in laser-driven systems.

In the vicinity of $\omega_{c}$, moreover, the $1 s \rightarrow 2 s, 2 p^{-}, 2 p_{z}$, $3 d^{-2}$, and $4 f^{-3}$ transition energies with (solid curve) and without (thin dashed curve) polaron correction exhibit a pronounced dip at $\omega_{c}$ and their amplitude decrease with increasing $F_{0}$ as shown in Figs. 12 and 13. This is due to the fact that both the binding energy and the MPC have a similar dip at $\omega_{c}$ and the amplitude of this dip for the MPC decreases with increasing $F_{0}$ and, importantly, in the vicinity of $\omega_{c}$ the dependence of the binding energy for the $1 s$ state on the laser field intensity is stronger than that of the other states as seen in the bottom of Fig. 7. For the same reason, the $1 s \rightarrow 2 p^{+}$transition energy with (solid curve) and without (short dashed-dotted curve) the effects of photon process also present an appreciable dip at $\omega_{c}$ when $F_{0}=1 \mathrm{kV} / \mathrm{cm}$ as shown in Fig. 12(a) since the effects of the photon process on the MPC for the $2 p^{+}$state are not clearly seen in Fig. 10(a).

More interestingly, with further increase of $F_{0}$, this dip disappears at $\omega_{c}$ for the $1 s \rightarrow 2 p^{+}$transition energy without the effects of photon process as shown in Figs. 12(b) and 13. The reason is that both the binding energy and the MPC for the $1 s$ and $2 p^{+}$states have almost the same dependence on the magnetic field in the vicinity of $\omega_{c}$. On the contrary, the $1 s \rightarrow 2 p^{+}$transition energy including the effects of the single-photon process presents a dip at $\omega_{c}$ and its amplitude increases with increasing $F_{0}$ as seen in Figs. 12(b) and 13, which is a consequence of the fact that the MPC with photon process to the $2 p^{+}$state exhibits an appreciable peak at $\omega_{c}$ and its amplitude increases with $F_{0}$ as shown in Fig. 10(b).

It is worth to note that, in order to obtain quantitative agreement between theoretical and experimental results, one needs to take into account the ITLF effects on the shallowimpurity states under the ITLFs and magnetic fields [17-23]. 


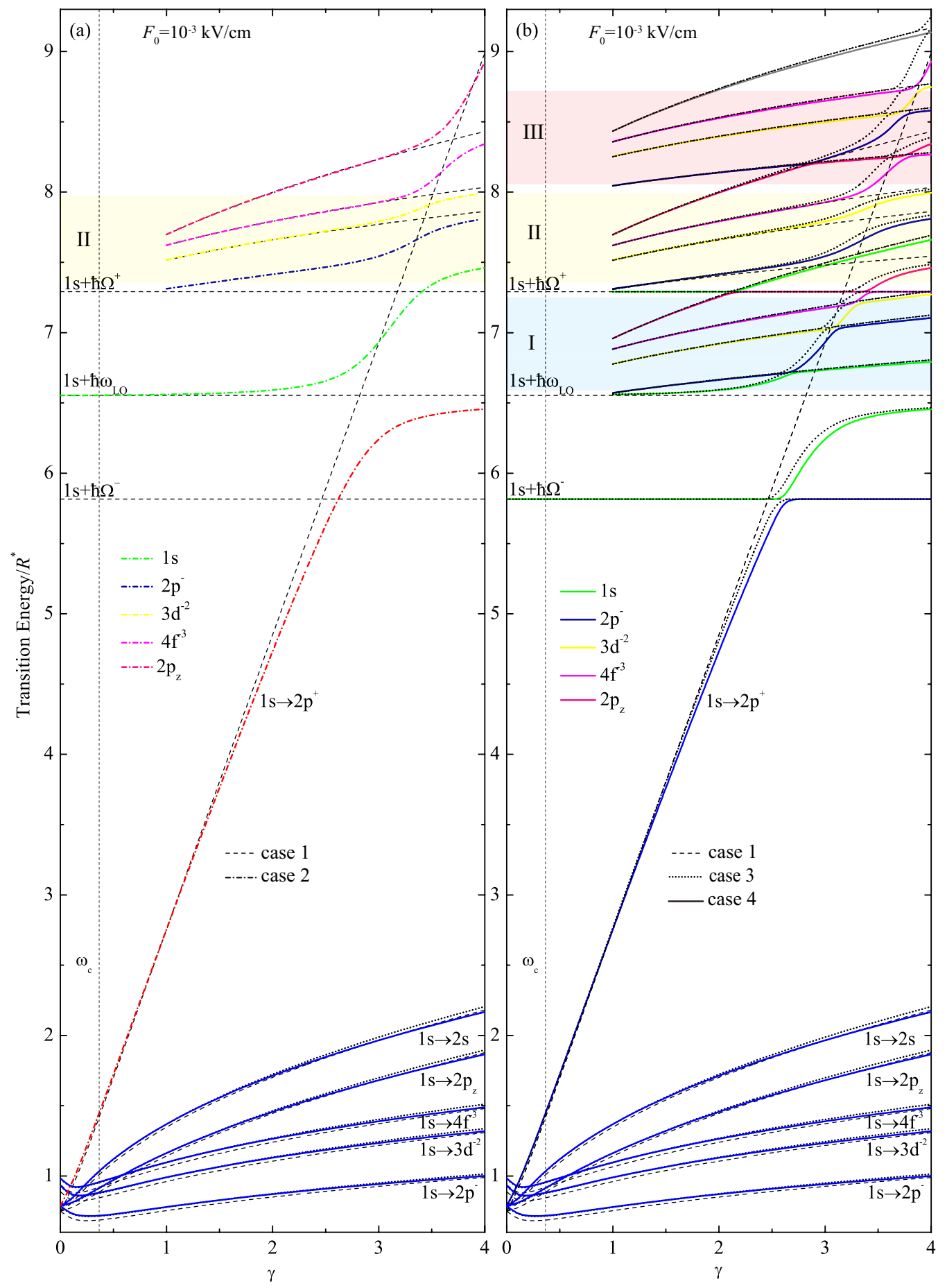

FIG. 11. Transition energy as a function of the magnetic field for several major impurity transitions at fixed $f=1 \mathrm{THz}$ and $F_{0}=10^{-3} \mathrm{kV} / \mathrm{cm}$ when the RMPE accompanied by (a) the elastic-photon scattering (region II) and (b) the emission (region I) and absorption (region III) of single photon. For comparison, we consider the following cases: (1) with the effects of single-photon process (thin dashed curve); (2) with the effects of polaron and band nonparabolicity (short dotted-dashed curve); (3) with the effects of polaron and single-photon process (short dotted curve); and (4) with the effects of polaron, single-photon process, and band nonparabolicity (solid curve). Here, $\hbar \Omega^{ \pm}=\hbar \omega_{\mathrm{LO}} \pm \hbar \omega$ and $\omega_{c}$ indicates the position of the cyclotron frequency.

Since there are at present no experimental investigations about the ITLF effects on shallow-impurity states in doped semiconductors and related nanostructures within the Faraday configuration, we are currently not able to compare our results with experiment. However, the current genera- tion of FELs can provide intense laser fields in the $\mathrm{THz}$ bandwidth, which makes our theoretical results testable. We thus hope that the theoretical findings discussed in this paper can be verified experimentally in the near future. 


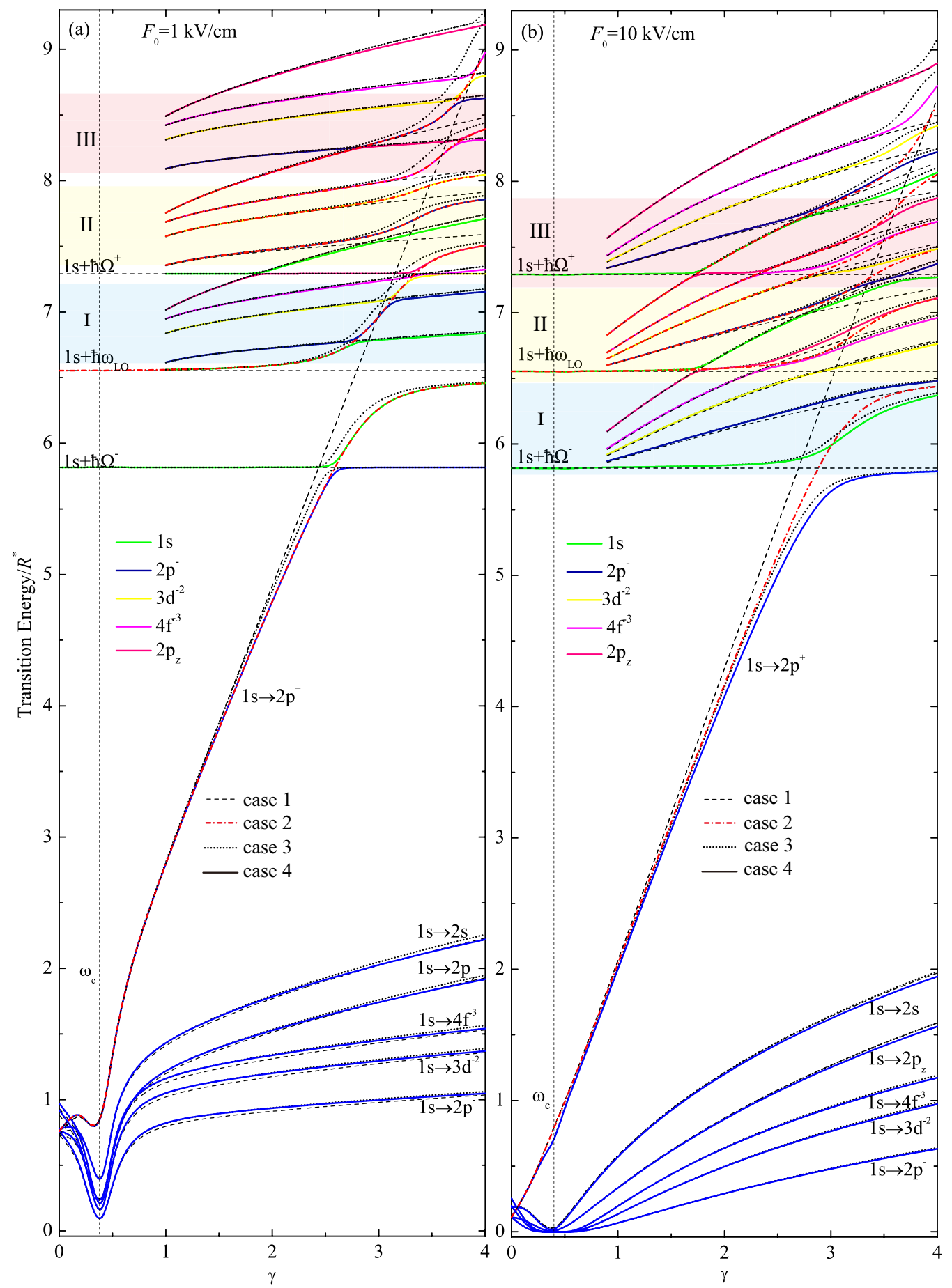

FIG. 12. Transition energy as a function of the magnetic field for several major impurity transitions at fixed $f=1 \mathrm{THz}$ for $(\mathrm{a}) F_{0}=1 \mathrm{kV} / \mathrm{cm}$ and (b) $F_{0}=10 \mathrm{kV} / \mathrm{cm}$ when the RMPE accompanied by the elastic-photon scattering (region II) and by the emission (region I) and absorption (region III) of single photon. For comparison, we consider the following cases: (1) with the effects of single-photon process (thin dashed curve); (2) with the effects of polaron and band nonparabolicity (short dotted-dashed curve); (3) with the effects of polaron and single-photon process (short dotted curve); and (4) with the effects of polaron, single-photon process, and band nonparabolicity (solid curve). Here, $\hbar \Omega^{ \pm}=\hbar \omega_{\mathrm{LO}} \pm \hbar \omega$ and $\omega_{c}$ indicates the position of the cyclotron frequency.

\section{CONCLUSIONS}

In this study, within the Faraday configuration, we have presented a theoretical study of the spectrum of shallowimpurity states in GaAs semiconductors in the presence of the ITLFs and magnetic fields. Within the effective-mass and dipole approximations, the ITLF effects on shallow-impurity states have been considered within a nonperturbative scheme in which the ITLF effects are exactly taken into account through the LdCP for electron-impurity coupling and the LdIP for e-p coupling. On this basis, we have used the variational 


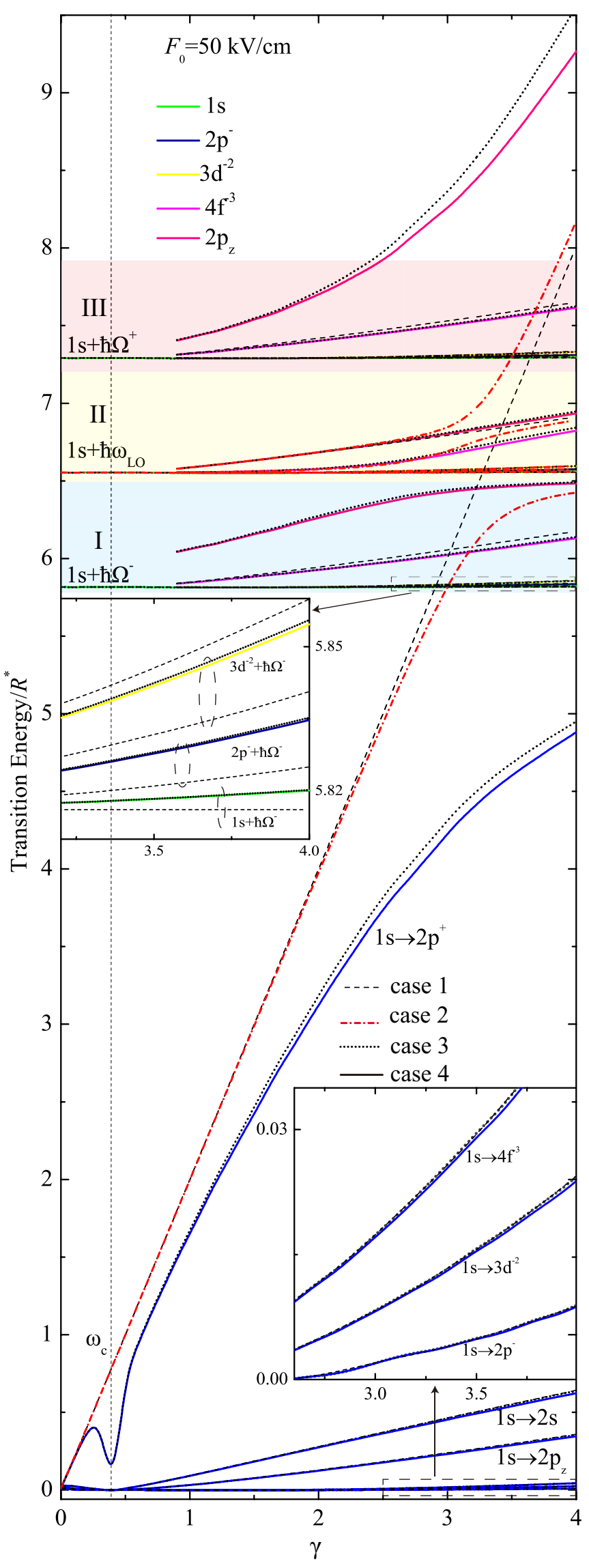

FIG. 13. The same as Fig. 12 for the laser field intensity $F_{0}=$ $50 \mathrm{kV} / \mathrm{cm}$. The marked areas are enlarged in the insets. method to investigate the influence of the external fields on the energy levels of shallow-impurity states and applied the TdPT to the theoretical study of the RMPE accompanied by the emission and absorption of photons. In conjunction with optical measurements, the binding energy and the internal transition energy including the effects of polaron, photon process, and band nonparabolicity have been calculated and discussed. The main conclusions obtained from this study are summarized as follows.

We found that the binding energy can be effectively modulated to be larger or smaller than the static binding energy by changing the ITLF at fixed magnetic field and vice versa, which is due to the fact that the $\mathrm{LdCP}$ can be enhanced or weakened with an appropriate choice of the two external applied fields, so that these intriguing behaviors can be understood in terms of electron-localization properties in this laser-driven systems. Moreover, a pronounced peak or dip can be observed at $\omega_{c}$ with a proper choice of the laser field intensity. These behaviors of the binding energy can be only observed in the Faraday configuration, which stems from the direct mutual coupling between vector potentials induced by the ITLF and magnetic field, respectively.

In the nonresonant polaron region, we found that the MPC to the $1 s, 2 s, 2 p^{-}, 2 p_{z}, 3 d^{-2}$, and $4 f^{-3}$ states can not only increase but also decrease with respect to the static MPC by varying the laser field intensity at fixed laser field frequency, which is physically consistent with the variation of the binding energy with magnetic field for different laser field intensities. On the contrary, the MPC with the effects of single-photon process to the $2 p^{+}$state increases with increasing $F_{0}$ since more channels are opened up in this case. Moreover, the MPC with photon process exhibits a pronounced peak at $\omega_{c}$ and its amplitude increases with increasing $F_{0}$, which is opposite to that of the other states.

In the resonant polaron region, 10 additional branches of the $1 s \rightarrow 2 p^{+}$transition were found which stem from the virtual interaction with the LO phonons accompanied by the emission and absorption of photons. Moreover, these additional branches of the $1 s \rightarrow 2 p^{+}$transition can be modulated to be far away from the reststrahlen band by varying the radiation frequency and an appreciable characteristic anticrossing behavior can be observed by properly choosing the laser field intensity and frequency, which indicates that the RMPE accompanied by the emission and absorption of photons can be observed experimentally and, in turn, provides a method to measure the e-p interaction in laser-driven systems.

The method used in this paper is capable of describing the correct behavior of shallow impurities in semiconductors and related nanostructures under the ITLFs and magnetic fields within the Faraday configuration, which provides a useful tool to reveal the intriguing properties of semiconductor systems under the two external applied fields, giving a degree of freedom that can be used in device applications.

\section{ACKNOWLEDGMENTS}

This work was supported by the National Natural Science Foundation of China (Grants No. 11404214 and No. 11455015) and the China Scholarship Council (CSC), 
Anhui Provincial Natural Science Foundation (Grant No. 1408085QA13), Key Projects of Anhui Provincial Department of Education (Grants No. KJ2017A406 and No. KJ2017A401). B.V.D. was financially supported by the Research Science
Foundation-Flanders (FWO-Vl) through a postdoctoral fellowship and M.V.d.D. was financially supported by the Research Science Foundation-Flanders (FWO-Vl) through a doctoral fellowship.
[1] D. Neamen, Semiconductor Physics and Devices (McGraw-Hill, New York, 2002).

[2] See, e.g., V. J. Goldman, H. D. Drew, M. Shayegan, and D. A. Nelson, Phys. Rev. Lett. 56, 968 (1986); H. Momose, H. Okai, H. Deguchi, N. Mori, and S. Takeyama, Phys. E (Amsterdam) 32, 309 (2006).

[3] C. B. Duke, G. G. Kleiman, and T. E. Stakelon, Phys. Rev. B 6, 2389 (1972).

[4] See, e.g., R. Cohen, J. Lodenquai, and M. Ruderman, Phys. Rev. Lett. 25, 467 (1970); M. V. Ivanov and P. Schmelcher, Phys. Rev. A 61, 022505 (2000).

[5] W. C. Henneberger, Phys. Rev. Lett. 21, 838 (1968).

[6] M. Gavrila and J. Z. Kaminski, Phys. Rev. Lett. 52, 613 (1984).

[7] F. Ehlotzky, Can. J. Phys. 63, 907 (1985); Phys. Lett. A 126, 524 (1988).

[8] H. S. Brandi, A. Latgé, and L. E. Oliveira, Phys. Status Solidi B 210, 671 (1998); Phys. Rev. B 64, 233315 (2001); 64, 035323 (2001); 70, 153303 (2004).

[9] For recent developments, see, e.g., A. Radu and E. C. Niculescu, Phys. Lett. A 374, 1755 (2010); S. Wei, Y. Zhu, and C. Xia, Superlatt. Microstuct. 49, 400 (2011); C. Gonźalez-Santander, T. Apostolova, and F. Domínguez-Adame, J. Phys.: Condens. Matter. 25, 335802 (2013); U. Yesilgul, Phys. E (Amsterdam) 74, 34 (2015); D. Laroze, M. Barseghyan, A. Radu, and A. A. Kirakosyan, Phys. B (Amsterdam) 501, 1 (2016); E. Kasapoglu, S. Sakiroglu, I. Sökmen, R. L. Restrepo, M. E. Mora-Ramos, and C. A. Duque, Opt. Mater. 60, 318 (2016); E. C. Niculescu, Chem. Phys. 487, 16 (2017).

[10] M. Pont, N. R. Walet, M. Gavrila, and C. W. McCurdy, Phys. Rev. Lett. 61, 939 (1988); M. Pont, N. R. Walet, and M. Gavrila, Phys. Rev. A 41, 477 (1990).

[11] M. Gavrila, J. Phys. B: At., Mol. Opt. Phys. 35, R147 (2002).

[12] F. M. S. Lima, O. A. C. Nunes, M. A. Amato, A. L. A. Fonseca, and E. F. da Silva, Jr., J. Appl. Phys. 103, 113112 (2008).

[13] M. Pont and M. Gavrila, Phys. Rev. Lett. 65, 2362 (1990); M. Dörr, R. M. Potvliege, D. Proulx, and R. Shakeshaft, Phys. Rev. A 43, 3729 (1991); F. M. S. Lima, M. A. Amato, L. S. F. Olavo, O. A. C. Nunes, A. L. A. Fonseca, and E. F. da Silva, Jr., Phys. Rev. B 75, 073201 (2007).

[14] A. L. A. Fonseca, M. A. Amato, and O. A. C. Nunes, Phys. Status Solidi B 186, K57 (1994); J. L. Nie, W. Xu, and L. B. Lin, Int. J. Mod. Phys. B 17, 2487 (2003).

[15] Q. Fanyao, A. L. A. Fonseca, and O. A. C. Nunes, Phys. Rev. B 54, 16405 (1996); J. Appl. Phys. 82, 1236 (1997).

[16] Y. P. Varshni, Superlatt. Microstuct. 30, 45 (2001).

[17] H. S. Brandi, A. Latgé, and L. E. Olieira, Solid State Commun. 107, 31 (1998).

[18] H. Sari, E. Kasapoglu, and I. Sökmen, Phys. Lett. A 311, 60 (2003).

[19] C. A. Duque, M. E. Mora-Ramos, E. Kasapoglu, H. Sari, and I. Sökmen, Eur. Phys. J. B 81, 441 (2011).

[20] E. C. Niculescu, Eur. Phys. J. B 85, 66 (2012).
[21] E. C. Niculescu and L. Burileanu, J. Optoelectron. Adv. Mater. 9, 2713 (2007); E. C. Niculescu and A. M. Radu, ibid. 9, 3598 (2007).

[22] F. E. López, E. Reyes-Gómez, N. Porras-Montenegro, H. S. Brandi, and L. E. Oliveira, J. Phys.: Condens. Matter 22, 045303 (2010).

[23] H. S. Brandi, A. Latgé, and L. E. Olieira, J. Appl. Phys. 92, 4209 (2002).

[24] See, e.g., A. Dousse, L. Lanco, J. J. Suffczyński, E. Semenova, A. Miard, A. Lemaître, I. Sagnes, C. Roblin, J. Bloch, and P. Senellart, Phys. Rev. Lett. 101, 267404 (2008); L. Schneebeli, M. Kira, and S. W. Koch, ibid. 101, 097401 (2008).

[25] F. E. López, E. Reyes-Gómez, N. Porras-Montenegro, H. S. Brandi, and L. E. Oliveira, J. Phys. D: Appl. Phys. 42, 115304 (2009).

[26] E. Kasapoglu, H. Sari, M. Güneş, and I. Sökmen, Surf. Rev. Lett. 11, 403 (2004).

[27] W. Wang, L. Xu, B. Wu, S. Zhang, and X. Wei, Phys. B (Amsterdam) 521, 122 (2017).

[28] See, e.g., D. M. Larsen, Phys. Rev. B 30, 4595 (1984); U. Merkt, M. Horst, T. Evelauer, and J. P. Kotthaus, ibid. 34, 7234 (1986); X. Wu, F. M. Peeters, and J. T. Devreese, ibid. 34, 2621 (1986).

[29] See, e.g., H. Fröhlich, Adv. Phys. 3, 325 (1954); R. P. Feynman, Phys. Rev. 97, 660 (1955).

[30] J.-P. Cheng, B. D. McCombe, J. M. Shi, F. M. Peeters, and J. T. Devreese, Phys. Rev. B 48, 7910 (1993); J. M. Shi, F. M. Peeters, and J. T. Devreese, ibid. 48, 5202 (1993).

[31] J. M. Shi, F. M. Peeters, J. T. Devreese, Y. Imanaka, and N. Miura, Phys. Rev. B 52, 17205 (1995); M. Grynberg, S. Huant, G. Martinez, J. Kossut, T. Wojtowicz, G. Karczewski, J. M. Shi, F. M. Peeters, and J. T. Devreese, ibid. 54, 1467 (1996).

[32] A. Wysmołek, R. Stȩpniewski, M. Potemski, B. ChwaliszPiętka, K. Pakuła, J. M. Baranowski, D. C. Look, S. S. Park, and K. Y. Lee, Phys. Rev. B 74, 195205 (2006).

[33] B. A. Mason and S. Das Sarma, Phys. Rev. B 33, 8379 (1986); C. D. Hu and Y.-H. Chang, ibid. 40, 3878 (1989); D. L. Lin, R. Chen, and T. F. George, ibid. 43, 9328 (1991); R. Chen, K. K. Bajaj, J. P. Cheng, and B. D. McCombe, ibid. 51, 9825 (1995); F. A. P. Osório, M. Z. Maialle, and O. Hipólito, ibid. 57, 1644 (1998).

[34] J. M. Shi, F. M. Peeters, G. Q. Hai, and J. T. Devreese, Phys. Rev. B 44, 5692 (1991); J. M. Shi, F. M. Peeters, and J. T. Devreese, ibid. 51, 7714 (1995); F. M. Peeters, J. M. Shi, and J. T. Devreese, Phys. Scr. T55, 57 (1994).

[35] S. Hameau, Y. Guldner, O. Verzelen, R. Ferreira, G. Bastard, J. Zeman, A. Lemaître, and J. M. Gérard, Phys. Rev. Lett. 83, 4152 (1999); S. Hameau, J. N. Isaia, Y. Guldner, E. Deleporte, O. Verzelen, R. Ferreira, G. Bastard, J. Zeman, and J. M. Gérard, Phys. Rev. B 65, 085316 (2002); B. S. Kandemir and A. Çetin, ibid. 65, 054303 (2002).

[36] R. M. O. Galváo and L. C. M. Miranda, J. Phys. C: Solid State Phys. 17, L41 (1984). 
[37] B. K. Meyer, D. M. Hofmann, D. Volm, W. M. Chen, N. T. Son, and E. Janzén, Phys. Rev. B 61, 4844 (2000).

[38] W. Xu, R. A. Lewis, P. M. Koenraad, and C. J. G. M. Langerak, J. Phys.: Condens. Matter 16, 89 (2004).

[39] W. Y. Wang and W. Xu, Phys. Rev. B 86, 045307 (2012).

[40] R. M. O. Galvão and L. C. M. Miranda, Am. J. Phys. 51, 729 (1983).

[41] R. Gargano, A. L. A. Fonseca, and O. A. C. Nunes, Phys. Rev. A 41, 2138 (1990); R. B. Pereira, A. L. A. Fonseca, and O. A. C. Nunes, J. Phys. Soc. Jpn. 67, 4098 (1998).

[42] C. Rodríguei-Cateilanos and M. T. Pérez-Maldonado, Superlatt. Microstuct. 27, 15 (2000); M. T. Pérez-Maldonado, C. Rodríguei-Cateilanos, and M. Sánchez-Gácita, Phys. Status Solidi B 232, 130 (2002).

[43] W. Xu and L. B. Lin, J. Phys.: Condens. Matter 13, 10889 (2001).

[44] C. A. S. Lima and L. C. M. Miranda, Phys. Rev. A 23, 3335 (1981); Phys. Lett. A 86, 367 (1981).

[45] L. C. M. Miranda, R. M. O. Galvãno, and C. A. S. Lima, Phys. Rev. B 28, 5313 (1983).
[46] L. C. M. Miranda, Phys. Lett. A 86, 363 (1981); N. S. Almeida and L. C. M. Miranda, Opt. Commun. 42, 241 (1982); T. C. Landgraf, J. R. Leite, N. S. Almeida, C. A. S. Lima, and L. C. M. Miranda, Phys. Lett. A 92, 131 (1982); C. A. S. Lima and L. C. M. Miranda, J. Chem. Phys. 78, 6102 (1983).

[47] T. Xu, L. Yuan, and J. Fang, Phys. B (Amsterdam) 404, 3445 (2009); W. Xie, Phys. E (Amsterdam) 43, 1411 (2011); 43, 1704 (2011); B. Vaseghi, G. Rezaei, and T. Sajadi, Phys. B (Amsterdam) 456, 171 (2015).

[48] J. J. Sakurai, Modern Quantum Mechanics (Pearson Education, Inc., San Francisco, 1994).

[49] P. M. Platzman, Phys. Rev 125, 1961 (1962).

[50] D. R. Cohn, D. M. Larsen, and B. Lax, Phys. Rev. B 6, 1367 (1972).

[51] T. Ruf and M. Cardona, Phys. Rev. B 41, 10747 (1990).

[52] F. M. Peeters, X. G. Wu, J. T. Devreese, C. J. G. M. Langerak, J. Singleton, D. J. Barnes, and R. J. Nicholas, Phys. Rev. B 45, 4296 (1992).

[53] P. W. Barmby, J. L. Dunn, C. A. Bates, and T. O. Klaassen, Phys. Rev. B 54, 8566 (1996). 